\title{
Thermo-Economic Optimization of Solar Thermal Devices by Coherent Integration of Technologies
}

\author{
Javier Cano Nogueras \\ Escuela Técnica Superior de Ingenieros Industriales, Universidad Politécnica de Madrid (UPM), Madrid, Spain \\ Email: javier.cano.nogueras@alumnos.upm.es
}

How to cite this paper: Cano Nogueras, J. (2020) Thermo-Economic Optimization of Solar Thermal Devices by Coherent Integration of Technologies. Energy and Power Engineering, 12, 671-707.

https://doi.org/10.4236/epe.2020.1211040

Received: October 12, 2020

Accepted: November 24, 2020

Published: November 27, 2020

Copyright (๑) 2020 by author(s) and Scientific Research Publishing Inc. This work is licensed under the Creative Commons Attribution International License (CC BY 4.0).

http://creativecommons.org/licenses/by/4.0/

\section{(c) (i) Open Access}

\begin{abstract}
Radiation is a form of energy where the angular variable of the direction of its photons has a primary importance, particularly for radiation concentration processes, which are essential tools to reach high temperatures from radiation beams (as the solar ones) with moderate intensities. Solar radiation cannot be used directly to feed thermodynamic cycles, and optical concentration must be applied to that goal. In general, reflection from mirrors is preferred to refraction by lenses in this case, because they have less optical aberrations. Concentration conveys very high temperatures in the receiver. However, the higher the temperature, the lower the efficiency of the solar thermal apparatus. Besides that, economy also suffers quite a lot when going to very high concentration factors, which is one of the main burdens in the development of Solar Thermal Energy. A new configuration of solar radiation concentrator is presented. It includes a salient innovation in the way the mirrors are given the right curvature by mechanical forces. Those mirrors are originally flat and do not need any special thermal treatment for this purpose. The whole device concept has been guided by the principle of thermo-economic coherence, which requires similar efforts in all degrees of freedom that have strong influence in the performance and cost of the system. The paper shows the decision tree that has oriented the project, following the principle of equilibrium in efforts, which leads to a design window of moderate values in the main variables. The prototype of this new configuration has already been built, and the first stage of research is considered to be finished, because the prototype has shown excellent conditions to include selected (fitting) technologies at a very low cost.
\end{abstract}

\section{Keywords}

Thermal Systems, Thermoeconomics, Optimization, Concentrated Solar 
Power, Azimuth Rotatory Solar Concentrator, New CSP Fresnel Concept, Prototype

\section{Introduction and Background}

Solar energy is the largest source of energy in our planet, by far and large. Note that fossil fuels were made by solar power captured by plants and the like many millions of years ago, and current wind energy is just a mechanical product from the interaction of current solar radiation with the atmosphere [1].

The average value of solar power reaching sea level is about 130,000 TW [2] which is 10,000 times as large as the human demand of energy.

As a source of energy, it has a main drawback-a somehow low power intensity, which implies moderate temperatures when interacting with objects. This fact is due to the Sun-Earth distance that conveys a reduction in the flux of photons (per square meter). In average, the power intensity of solar radiation in the outer atmosphere is $1.35 \mathrm{~kW} / \mathrm{m}^{2}$ [3], and it goes down to 1 when it reaches sea level, presuming a clear sky. Clouds reduce to zero the intensity of direct radiation (coming directly from the Sun) although diffuse radiation scattered from the clouds is still useful to see.

Radiation is a form of energy where the angular variable of the direction of its photons has a primary importance, particularly for radiation concentration processes, which are essential tools to reach high temperatures from solar direct radiation. Diffuse radiation cannot be concentrated, because of the geometric laws of radiation interaction with different types of bodies.

Two mechanisms can be used for that purpose: refraction (lenses, as in Galileo's telescope) and reflection (mirrors, as in Newton's telescope). Curiously enough, refraction is preferred in radiation concentration for Photo-Voltaic applications [4], while reflection dominates solar thermal applications.

Concentration by reflection is restricted by Liouville theorem [5] on particle beams, which states that the emittance of a beam cannot decrease. It seems to be a purely geometric theorem, but it has a clear connection with entropy, because the emittance of beam is a measure of its entropy, and decreasing the emittance would be the same as reducing entropy by spontaneous mechanisms, which is something ruled out in our Physics. For instance, the aperture angle of a radiation beam coming directly from the Sun is 9 milliradians, but it jumps to 15 and even 25 after being reflected by the concentrating mirrors. Of course, the geometrical overlapping of the imprint of different beams on a given spot, produces an actual multiplication of the power intensity, or solar flux, by a factor of 50 , 100, 1000 and more. (The theoretical limit, 40,000 in round numbers, can be computed by two totally different ways, which have the same root: it can be made by geometric considerations on a perfect parabolic cup; or by pure Thermodynamics, stating that the maximum concentration cannot produce a tem- 
perature (in a pure black body absorbing the concentrated radiation) higher than temperature of Sun's surface (5760 K). Nevertheless, usual concentration factors remain below 100).

If our engineering objective is to absorb solar heat for feeding a thermodynamic cycle [6], high temperatures are needed, as prescribed by Carnot's theorem. This conveys higher concentration factors, that will require better or more powerful concentration apparatus. This is one of the main challenges in Solar Thermal Energy Engineering, for not to say the most important. This paper addresses this challenge from a very particular viewpoint, stemming from the experience of the different types on concentrator modules that have tested and applied in last 40 years.

It is worth noting that a relevant problem in radiation concentration engineering is the very large surfaces required to reach high power levels. This fact implies huge amounts of structural material, plus mirrors, plus pipes, and so forth ([7] [8]). A $50 \mathrm{MW}$ solar thermal plant can require an amount of concrete ten times as large as the total concrete required in a 1000 MW Nuclear Power Plant. However, safety issues and sustainability principles point out that energy research must be focused onto renewable energies, which sets up an important technical challenge, which is the final object of this research.

An elementary requirement from Carnot's theorem is the need of very high temperatures in the heat carrier fluid of the solar plant. However, the higher the temperature, the lower the efficiency of the solar thermal apparatus, because thermal losses increase quite a lot. Besides that, economy also suffers when going to very high concentration factors, which is one of the main burdens in the development of Solar Thermal Energy (also known as CSP: Concentrating Solar Power). This fact sets up a challenge to researchers in this field, which still is very open and needs to reduce cost by a massive optimization of the system. This is the point where Coherent Integration of Technologies plays a major role, as explained in this paper. From fixed to rotatory structures, to planar combed mirrors, to Sun tracking sets, to piping system, to absorbing coatings, and so forth, make a puzzle where solutions must be generated by the very process of analyzing technology alternatives in a coherent and self-consistent way, without forgetting the entropy generated by uncertainties and allowances in the real world. Optimization seems in many cases a mathematical process ([9] [10]), but its equations are full of coefficients representing technology characteristics, and they are as dominant as the equation themselves. Therefore, Coherent Integration of Technologies must have a fundamental role in solar thermal energy development.

An introductory example is given in Figure 1 and Figure 2 where an original solar thermal prototype is shown. Its structure corresponds to a coherent integration off technologies, where each device off the full machine must be optimized twofold: internally, as a component with a given functionality, and externally, to match the features of the system. 


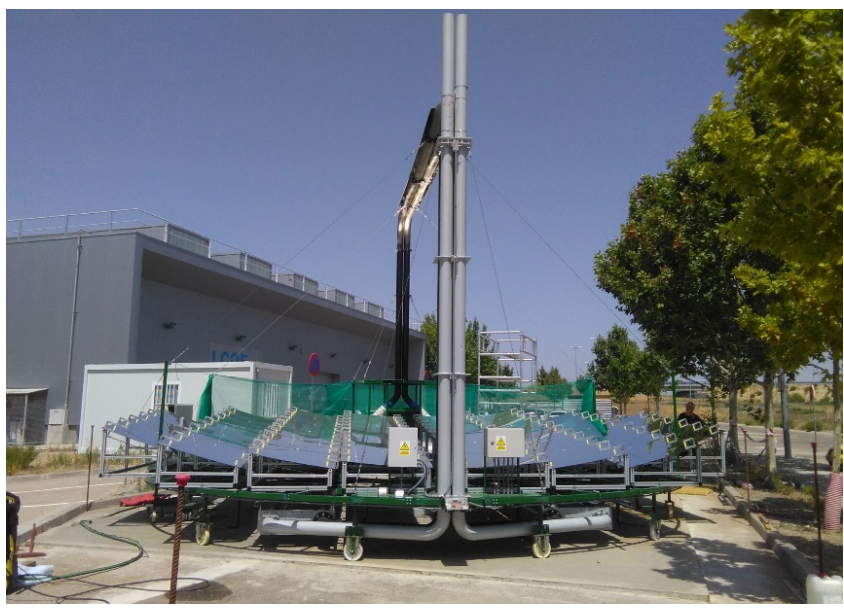

Figure 1. Full prototype view.
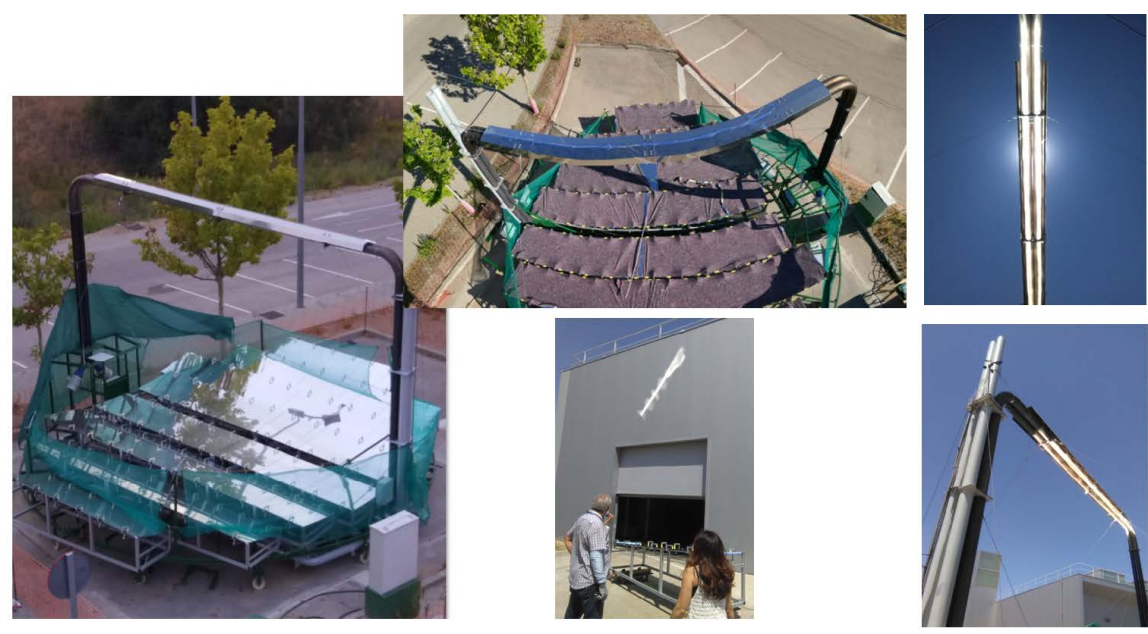

Figure 2. Different views from the constructed prototype.

In the following section, a brief summary on the structure of a Solar Thermal Power plant is presented, in order to have a reference of the technical features and functionalities of the different parts of that system. In particular, it will be needed in section 3, devoted to the "thermal coherence" methodology, which will be the tool to justify the need of a totally new and comprehensive approach in the design and operation of a solar power plant. Such a new family of plants, called "Sundial", is introduced in section 4. One of its key points is that mirrors are fixed in relation to the receiver, although all these elements are mounted on a spinning platform (turning at the same speed as the apparent speed of Sun in the local sky of the plant). Section 5 deals with the actual embodiment of the prototype, where the different technologies chosen to build the apparatus had to match. The concept of Coherent Integration of Technologies appears as a guide to take appropriate decisions. Both this concept and the Sundial design still have a long way to be studied. Section 6 shows an example of how the device works through the temperature data measured on the receiver and a brief discussion about the results, and finally section 7 deals with conclusions and future work of 
the proposed methodology and Sundial prototype constructed.

\section{Structure of a Solar Thermal Power Plant}

A solar thermal power plant is made up of the systems described in the following list, which make up what we can call a thermal energy chain. It is obvious that the systems in that chain must be correctly connected to each other. This requirement is important and affects both the total "captured heat" and its temperature [11]. In Thermal Engineering, this is presented by exergy analysis, which is an aid to STE plant design [12]. The list is as follows:

- Mirror field, concentrator: original sunrays are reflected to a focal zone, where radiation reaches much higher intensities than the value of the original solar radiation, which will be around $1 \mathrm{~kW} / \mathrm{m}^{2}$ in very good atmospheric conditions. The "concentration factor" is the parameter that characterizes the optical performance of the concentrator.

- Receiver, or thermal assembly, which is a tube or a bundle of tubes that can have very different geometries, where a (high) fraction of the thermal energy of the concentrated radiation is captured by the heat carrier fluid that flows inside the tube or tubes. The shape and size of the receiver, as well as the flow pattern in its tubes, are essential elements for the design (to reduce thermal losses, above all). The allowable material temperature is the main limiting factor of the receiver's performance. It is worth noting that thermal losses from the receiver increases with the operating temperature. This is another limiting effect.

- Heat transfer fluid piping. Solar thermal power plants are very large due to the low intensity of the original solar radiation and this make it necessary to have very long pipes to connect all the systems [13]. The pressure drop across the circuits can reach very high values, which leads to very high pumping power values (which can eventually be higher than the electrical power of the plant; a curious nonsense).

- Thermal Energy Storage (TES), which is not mandatory, but it can contribute quite a lot to the get the best from the potential of these plants [14]. There is a variety of configurations and materials for building a TES. Nevertheless, molten salts seem the most suitable for current investments, although they present important drawbacks, as the risk of solidifying at relatively high temperatures. It goes without saying that charging and discharging the storage implies some loss of thermal energy, and a decrease in temperature.

- Block of Power (BOP): The heat carrier fluid piping channelizes most of the thermal energy captured to the block of power, where it is delivered to a thermodynamic cycle. The most popular one nowadays is the standard steam Rankine cycle, although some proposals have been made to use the JouleBrayton cycle. It is also worth remembering that the cycle efficiency increases with the temperature of the hot focus (which is the hottest flow of the heat carrier fluid). 
It was already said that thermal losses from the collectors increase as the operating temperature increases, and this is particularly true for high $T_{h}$ values, because radiation losses depend on the fourth power of $T$. A receiver's efficiency can be defined as the ratio between the thermal power captured by the heat carrier fluid and the total thermal power arriving to the receiver as concentrated radiation. This efficiency is practically one for $T_{h}=T_{c}$ and goes down asymptotically to zero as $T_{h}$ increases.

The product of both efficiencies is zero both for $T_{h}=T_{c}$ and for $T_{h}$ going to infinity. Therefore, somewhere in the middle there will be a maximum, and this is the goal of the designers, although other factors must also be considered, as material behavior under different conditions. For instance, the selective coating where the concentrated radiation impinges on, can undergo degradation at very high temperatures.

There are several alternatives for selecting the type of concentrator and the type of receiver, although both elements are so closely connected that they can be considered as a single unit. An essential factor in this coupling is solar tracking, which is the aggregation of hardware and software to follow the sun for producing the sought reflection onto the receiver. The final outcome of this set of technical tasks is the capture of solar energy into a thermodynamic cycle to generate electricity, although other endothermic applications could be used, as the production of synthetic fuel (artificial methanol, for instance).

Within this context, the analysis on the solar domains of the plant must be carried out with an appropriate tool to embody both mathematical foundations of optimization and practical restrictions from technology. It is worth underlining that optimum values of relevant variables will depend quite a lot on the technology used in each part of the solar plant. And it is also obvious that fitting technologies must have a similar degree of perfection. It is usually said that the weakness of a chain is that of its weakest block. It is out of question putting a Formula 1 engine into a compact car, and thermal coherence methodology is a mathematical tool to avoid those mismatches by using appropriate functions to characterize the thermal performance of the parts of the system, and their cost, which is the subject of next section.

\section{Thermo-Economic Coherence in Solar Thermal Facilities}

The concept of "thermal coherence" was introduced by Rovira and Martínez-Val in the paper [15], and it is a tool actually suited for Solar Thermal, because they can easily be decomposed in modules with clear functionality, which can be characterized by an "energy efficiency" and a unitary cost.

The following scheme shown in Figure 3 gathers the fundamental parts of CSP thermal coherence optimization. It summarizes the analytical steps that must be carried out in order to close an optimized design.

Indeed, the full process can be very long and can need lots of engineering resources, because a good optimization exercise would theoretically end with the 
identification of the best possible CSP embodiment, for a given scenario for CSP to be deployed.

The CSP Optimization scheme represents the options that has to be considered when designing the solar thermal plant. With all these elements in mind, we can reach the optimization of the plant design according to the tool and criteria set out in [15].

With all these steps we achieve the optimization of the design, but we still have the other part pending, the construction and therefore the integration of the design.

The construction of the solar thermal facility, our Sundial prototype, requires the coherent integration of numerous disciplines and technologies to get closer to the expected thermal performance resulting from applying the CSP optimization scheme.

The diagram of the Figure 4 shows a relationship of the main elements that are considered to transfer the optimized design to the construction of the prototype through the coherent integration of technology.

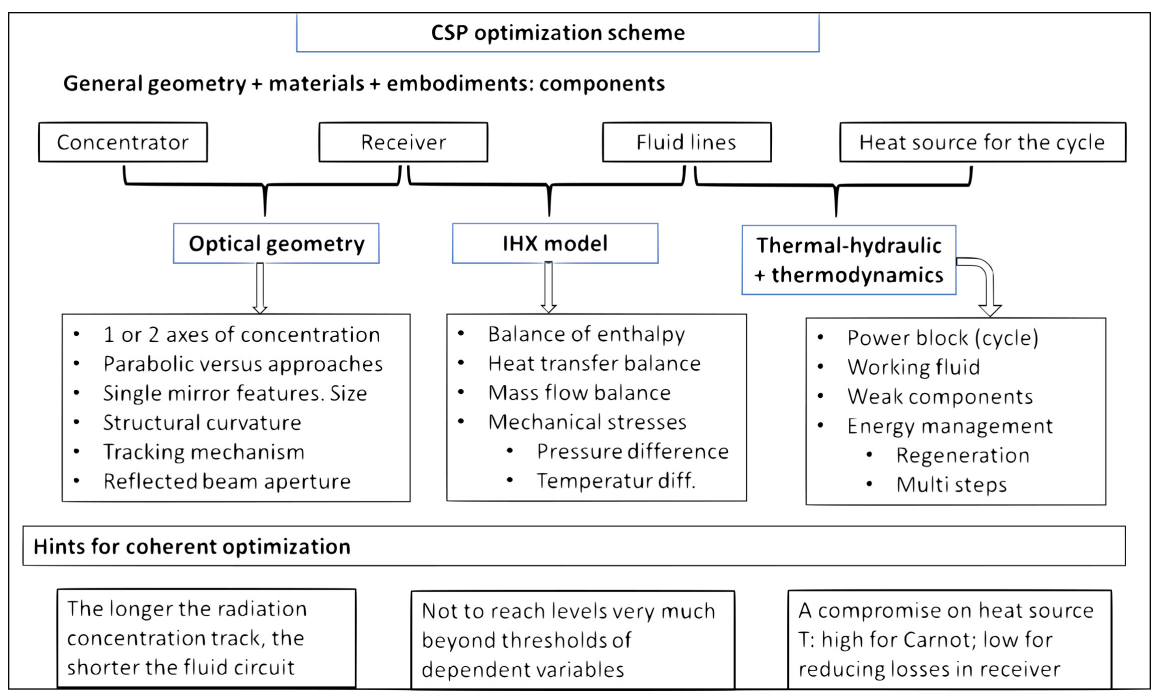

Figure 3. CSP Optimization scheme.

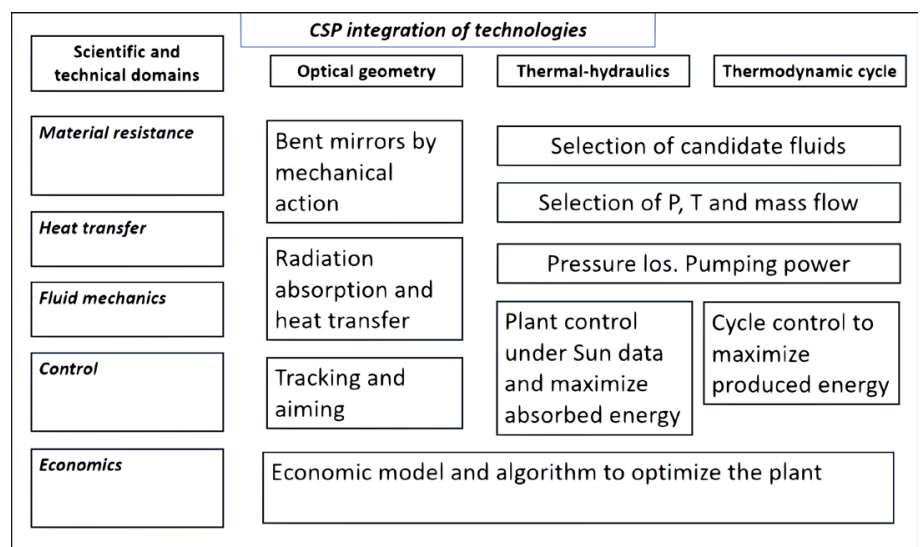

Figure 4. CSP integration of technologies. 


\subsection{Realistic Thermal Systems}

The approach developed to formally study a thermal system is based on splitting the case into two parts, so that the best relation between them can be identified. This approach can be applied to a complete system, as a coal-fired power plant or a solar thermal unit or can be applied to a subsystem of the plant.

In a solar thermal unit, the divided parts correspond to the Solar Field and the Generation Block. In a coal-fired power plant, the split parts would be the boiler and the turbine.

At a lower level, in the solar case we found that the Solar Field can be divided into two parts, the concentrator and the receiver.

We will examine firstly the general case with a boiler and a turbine, and we will use the same procedure later on, to study the concentrator and the receiver.

- The thermal generation block, where a source of heat is created from an original source of energy. (An efficiency law can be associated to this block, in relation to temperature: the higher $T$ values in the generation block, the higher the values of the thermal losses, because the $T$ gap between the conversion block and its surroundings will increase. Of course, thermal insulation can reduce this trend, of decreasing efficiency as $T$ increases, but this law exists for each given item in energy generation involving heat. At the same time, a cost law can be formulated to express that increasing operational $T$ in a given application, goes with a trend of increasing costs. Higher $T$ values require more expensive materials, for instance. Maintenance can be more difficult and maybe more frequent. In summary, as a general rule for well-behaved thermal devices, generation blocks show decreasing efficiencies and increasing costs as operational $T$ are raised).

- An energy conversion block, which embodies a thermodynamic cycle for converting the thermal energy into mechanical energy, and eventually into electricity. In this block, the rule for costs is like the previous one, for similar reasons. However, efficiency in conversion bocks are just the opposite, as it is perfectly known by Carnot's principle.

In most of current applications of thermal systems, the original source of energy is chemical combustion. Nuclear reactions, namely fission in current nuclear reactors, also generate heat. The same happens with electromagnetic microwaves, at a lower scale in energy exploitation.

In the field of electricity generation in the future, solar thermal power plants will have to play an important role for Energy Sustainability [16]. Besides that, they are a very good example of "Thermal system" and they can therefore be studied for the sake of featuring Thermal Systems and the need to have a good framework to establish their design windows. Of course, they are structured into two blocks:

- The solar collector, where solar radiation is collected as thermal energy of a heat carrier fluid. As a lot of collectors are usually necessary to reach a sizeable power, it is generally called "solar field". 
- A block of power (BOP) where energy from the heat carrier fluid is converted into mechanical energy in a thermal machine (either a turbine or an engine).

Collectors of the solar field (SF) are actually composed of two sub-systems:

- The concentrator, where direct solar radiation is concentrated onto a focal spot, either by reflection (mirrors) or refraction (lenses), although the former clearly dominate the field in Solar Thermal Power. In photovoltaic applications (PV) lenses are used more frequently. In both cases, high concentration factors can be achieved.

- The receptor, where concentrated solar radiation impinges on an absorbing surface, from which it is internally transferred to the heat carrier fluid, although a fraction of the thermal energy carried by the fluid leaks to the atmosphere or to the ground, or it is emitted by radiation from the receptor.

Although we can go into finer details of the system structure, the two-block structure is the right element for optimization analysis. Any block can afterwards be decomposed into two parts, and the analysis can be repeated in the corresponding scale, although macro conclusions are mainly derived from the first analysis in the two macro blocks already cited. The system is outlined in Figure 3, where a very important element is the heat carrier fluid, which is mainly characterized by its temperature $T$.

Energy, as an extensive variable, can be treated in a linearly scalable way, in a first approach, and the analysis can be done for a normalized power in a given part of the system, either at the starting point, $E_{0}$ or at the end as useful energy $E_{u}$. However, it is worth noting that "power" can be (and usually is) an important independent variable of the system, affecting the price of the components in a large extent. In general, specific investments $(\$ / \mathrm{kW})$ are cheaper as the components grow (in power, of course). So, a sound analysis would have to include this effect, which can be embodied in the calculations in several ways. The simplest one is to repeat calculations for several levels of power, so having a final map of the system performance in the full range of interest. For each case, the power value is fixed in a definite part of the system (the shaft of the turbine, for instance) and the cost is calculated on the unitary prices of components corresponding to that level. This approach has the advantage of a good accuracy, but its losses information on the stochastic distribution of some variables.

\subsection{Economics as a Base of Coherence Optimization}

Radiation is a form of energy where the angular variable of the direction of its photons has a primary importance, particularly for radiation concentration processes, which are essential tools to reach high temperatures from radiation beams (as the solar ones) with moderate intensities. Solar radiation cannot be used directly to feed thermodynamic cycles, and optical concentration must be applied to that goal. In general, reflection from mirrors are preferred to refraction by lenses in this case, because they have less optical aberrations, and are cheaper. 
Nevertheless, they are restricted by Liouville theorem on particle beams, which states that the emittance of a beam cannot decrease. The geometrical overlapping of the imprint of different beams on a given spot, produces an actual multiplication of the power intensity, or solar flux, by a factor of 50,100, 1000 and more. This fact conveys very high temperatures in the receiver. However, the higher the temperature, the lower the efficiency of the solar thermal apparatus. Besides that, economy also suffers quite a lot when going to very high concentration factors, which is one of the main burdens in the development of Solar Thermal Energy (also known as CSP: Concentrating Solar Power). This fact sets up a challenge to researchers in this field, which still is very open and needs to reduce cost by a massive optimization of the system.

The Exergy Method [17] is an alternative, relatively new technique based on the concept of exergy, loosely defined as a universal measure of the work potential or quality of different forms of energy in relation to a given environment.

[18] surveys briefly the recently developed methodologies that reveal the cost effectiveness of sought energy-resource-saving ideas by design and focuses on one methodology that became known as thermoeconomics.

Figure 5 show the block diagram of a solar power plant with two main parts, Solar Field and Block of Power. In our analysis there will a power $P_{0}$ representing the original solar energy arriving to the solar field, which produces a flow of heat carrier fluid with a temperature $T$, which is one of the two connecting variables with the second block. The other variable is the energy per second carried by the heat carrier fluid in the connection between blocks, $P$.

The first block, or Solar Field, is characterized by an efficiency " $P$ ", which a function of $T$. Similarly, the BOP will have an efficiency " $g(T)$ ", and it could be written:

$$
\begin{gathered}
P(T)=P_{0} \cdot f(T) \\
P_{u}(T)=P(T) \cdot g(T)=P_{0} \cdot f(T) \cdot g(T)
\end{gathered}
$$

At the same time, in order to find out the cost of each block, information will be needed on the micro-economics of the components and the macro-economics of borrowing money to make an investment. We presume those items are known, and costs can be calculated. They will be called " $S$ " for the solar field, and " $B$ " for the block of power (or Balance of Plant). In reality, the cited micro-economics and macro-economics are very important and deserve an appropriate explanation, because the total cost of the energy "treated" in each block is composed of different items, not only investment or initial cost, but O\&M (operation and maintenance), fuel (not in the solar case), insurance policies, and others. It is presumed that we know all these data and costs can be expressed as:

$$
\begin{gathered}
S\left(P_{0}\right)=P_{0} \cdot s(T) \\
B(P)=P \cdot b(T)=P_{0} \cdot f(T) \cdot b(T)
\end{gathered}
$$

where $s(T)$ and $b(T)$ are the specific costs of each block (\$/kW). 


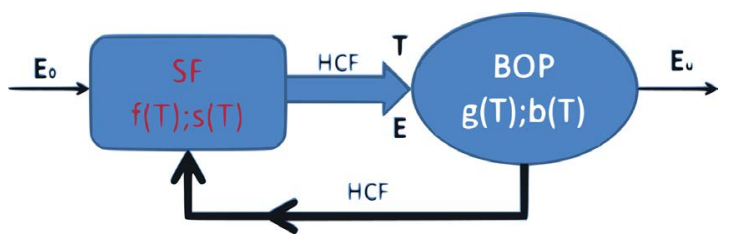

Figure 5. Block diagram of a solar power plant with two main parts: Solar Field and Block of Power. The heat carrier fluid (HCF) is another element of priority, and the most important variable to characterize the plant performance is the fluid temperature at the entrance of BOP from the SF.

Properly speaking, the economic analysis will not be done for an "instant" but for a period of time, namely one year. Investment cost must be actualized and shared among operation years. Most of the other costs are based on annual accounts. We consider that the plant will work several hours per year, at constant power. This is a strong simplification, and "time" has to be treated as an independent variables in any advanced analysis, but not in this case, where our attention should be fixed on $T$. So, the relevant balance must be expressed as

$$
\begin{gathered}
E(T)=E_{0} \cdot f(T) \\
E_{u}(T)=E(T) \cdot g(T)=E_{0} \cdot f(T) \cdot g(T) \\
S\left(E_{0}\right)=E_{0} \cdot s(T) \\
B(E)=E \cdot b(T)=E_{0} \cdot f(T) \cdot b(T)
\end{gathered}
$$

where the specific costs are expressed in $\$ / \mathrm{kWh}$ (in fact, it is used $\mathrm{c} \$ / \mathrm{kWh}$ or $\$ / M W h$, which are not equivalent). The total specific or unitary cost for Solar Thermal Plants were about $25 \mathrm{c} \$ / \mathrm{kWh}$ or $250 \$ / \mathrm{MWh}$ in values of 2010 from [19]. Approximately, 70\% - 75\% of it was from the SF (so, "s" was about $18-19$ $c \$ / \mathrm{kWh}$ ) and $20 \%-25 \%$ for the BOP (" $b$ " about $5-6 \mathrm{c} \$ / \mathrm{kWh}$ ) the rest of the cost corresponding to auxiliary buildings and others.

It should be noted that the blocks act as factors (multiplying) for the energy (or the power) but they are additive for the cost:

$$
C=S\left(E_{0}\right)+B(E)=E_{0} \cdot(s(T)+f(T) \cdot b(T))
$$

In a first approach we have considered that the system is fully linear with energy (or power) and $f, g, s$ and $b$ do not depend on $E_{0}$ or $E_{u}$, but the real fact is that they depend, because of scale economy of some manufacturing processes. A usual design practice is to fix a level of energy, or power, at a given point of the power chain, for instance $P_{u}$, which is the usual way for meeting requirements established by energy policy and planning. It is also possible to fix $E_{0}$, because this is given, in solar energy, by the amount of land available for the plant, and the filling factor specific of the collectors to be used (the filling factor being the fraction of ground covered by collectors). In our case, we shall consider we have a given value of primary energy, $E_{0}$, and it will be fixed.

As already said, $E_{0}$ or $E_{u}$ can be considered as independent variables in the problem, and therefore we would have to use partial derivatives for finding 
maxima or minima. An alternative way to use $f\left(T, E_{u}\right)$ and the like, is based on selecting a set of economic scenarios with different values for specific costs and efficiencies, and the optimization analysis is carried out for each scenario, so obtaining the required map of performances, costs and productivity.

With this background, equations can be analyzed to identify conditions or criteria leading to maxima or minima with special relevance in design decisions of thermal plants, particularly in the domain of Solar Thermal Energy.

\subsection{Optimization Study}

Productivity is defined by

$$
Y=\frac{E_{u}}{C}=E_{0} \cdot f(T) \cdot \frac{g(T)}{E_{0}} \cdot(s(T)+f(T) \cdot b(T))
$$

Considering the former hypothesis and methodology, these equations can be analyzed in order to identify conditions or criteria leading to maxima or minima with special relevance in design decisions of thermal plants, particularly

$$
\begin{gathered}
Y=f(T) \cdot \frac{g(T)}{s(T)+f(T) \cdot b(T)} \\
\frac{\mathrm{d} Y}{\mathrm{~d} T}=Y^{\prime}=\frac{\left(f^{\prime} \cdot g+g^{\prime} \cdot f\right) \cdot(s+f \cdot b)-\left(s^{\prime}+f^{\prime} \cdot b+b^{\prime} \cdot f\right) \cdot(f \cdot g)}{(s+f \cdot b)^{2}}
\end{gathered}
$$

Setting the derivative equal to 0 , it holds

$$
\begin{gathered}
Y^{\prime}=0 \\
\left(f^{\prime} \cdot g+g^{\prime} \cdot f\right) \cdot(s+f b)-\left(s^{\prime}+f^{\prime} \cdot b+b^{\prime} \cdot f\right) \cdot(f \cdot g)=0 \\
\frac{f^{\prime} \cdot g+g^{\prime} \cdot f}{f \cdot g}-\frac{s^{\prime}+f^{\prime} \cdot b+b^{\prime} \cdot f}{s+f \cdot b}=0
\end{gathered}
$$

which is in fact an equality between logarithmic derivatives

$$
\begin{aligned}
\frac{f^{\prime} \cdot g+g^{\prime} \cdot f}{f \cdot g} & =\frac{s^{\prime}+f^{\prime} \cdot b+b^{\prime} \cdot f}{s+f \cdot b} \\
\frac{\mathrm{d} \ln (f \cdot g)}{\mathrm{d} T} & =\frac{\mathrm{d} \ln (s+f \cdot b)}{\mathrm{d} T}
\end{aligned}
$$

Last two equations resemble notably to equations

$$
\frac{P^{\prime}}{P}=\frac{C^{\prime}}{C}
$$

and

$$
\frac{\mathrm{d}(\ln P)}{\mathrm{d} T}=\frac{\mathrm{d}(\ln C)}{\mathrm{d} T}
$$

for the single block system; where $P$ is a production function an $C$ is a cost function, and they convey the same conclusion: optimum in productivity, which is the situation of minimum cost of the generated energy, happens when the relative variation of production equals the relative increase of cost, and this equality 
will happen at a temperature, $T^{ \pm}$. As already said, before reaching this point $T^{ \pm}$, it is worth increasing the working temperature because the effect on production is bigger than the effect on cost. Beyond that point, the situation is just the opposite, and increasing $T$ entails higher costs which are proportionally bigger than the positive effect in production.

Apparently, the former condition for the maximum productivity does not embody any supplementary criterion on the individual specific values: $f, g, s$, and $b$; but it does. Namely, if blocks SF and BOP are not dimensioned in a coherent way, the productivity will suffer from a decrease of performance. Imagine, for instance, that we select the solar field SF for delivering oil at $800^{\circ} \mathrm{C}$ (value of $T$ ), which implies that radiation losses are going to be relevant, and therefore $f\left(800^{\circ} \mathrm{C}\right)$ will have a moderate value. In the second block, a Rankine cycle has been selected for BOP, with a Turbine Inlet Temperature of $400^{\circ} \mathrm{C}$, which conveys a limitation on the value of Carnot's efficiency, with a relatively moderate value of $g$. So, both efficiencies $f$ and $g$ will have smaller values than the case of having a $T$ value closer to the temperature needed to feed the Rankine cycle, for instance $450^{\circ} \mathrm{C}$ or $500^{\circ} \mathrm{C}$. This problem will be treated later.

There is another problem to be addressed first, which is the relation between the foregoing optimization, expressed as maximizing productivity, and another optimization, expressed as maximizing production, i.e., to maximize $E_{u}$ if $E_{0}$ is fixed; or the opposite, to minimize $E_{0}$ if $E_{u}$ is fixed. In any case, this is equivalent to maximize the function

$$
\begin{gathered}
Z=f \cdot g \\
Z^{\prime}=f^{\prime} \cdot g+f \cdot g^{\prime} \\
\frac{f^{\prime}}{f}=-\frac{g^{\prime}}{g}
\end{gathered}
$$

Which is

$$
\frac{\mathrm{d} \ln f}{\mathrm{~d} T}=\frac{\mathrm{d} \ln g}{\mathrm{~d} T}
$$

It is of primary interest to find out if this maximum, and the maximum of productivity, have any relation among them. Ideally, the best situation would be a coincidence between both maxima, because the system would reach its optimum value of production with the maximum productivity, or minimum cost per unit of production. If the right-hand-side member of Equation (19) is used in Equation (16), the criterion for coincidence is:

$$
\frac{s^{\prime}+f^{\prime} \cdot b+b^{\prime} \cdot f}{s+f \cdot b}=0
$$

This condition can be met by $(s+f \cdot b)$ going to infinity, but such a trivial condition on an infinite cost of the plant is out of practical meaning. The actual condition to be fulfilled is

$$
s^{\prime}+f^{\prime} \cdot b+b^{\prime} \cdot f=0
$$


This implies that the total cost of the plant is constant, as can be derived from Equation (9)

$$
C=S\left(E_{0}\right)+B(E)=E_{0} \cdot(s(T)+f(T) \cdot b(T))
$$

This is an external requirement with direct implications in internal parameters, namely $s, f$ and $b$. In classical theory of optimization, it could be treated by a Lagrange Multiplier. In current computational work, calculations could be carried out according to a systematic procedure, with cost splitting between the SF and BOP.

The procedure could start by choosing a very high value of the cost of SF, in relation to the total cost that must be kept constant, for instance, $90 \%$ of it. This assumption gives a vale of $s$, and an associated value of $f$, which means that $\mathrm{b}$ is computed as the number to arrive to the total cost $C$ (remember that a fixed value of $E_{0}$ has been chosen as a design parameter, in order to have a consistent series of cases).

Then, the fraction of $90 \%$ is decreased continuously for successive calculations, and it can finish whenever chosen by the designer. The outcome would be a series of cases with the same total cost and the same primary energy, $E_{0}$. The best case would be the one with maximum $E_{u}$.

As an alternative, $E_{u}$ can be fixed by the designer, and the best case would be the one with the minimum primary needs, $E_{0}$.

Indeed, the foregoing case is very interesting because it is realistic, in the sense that money is limited, and a constraint in that sense can be as simple as stating that total cost must be constant.

\subsection{Solar Thermal Principles for Coherence Optimization}

Thermal coherence implies a proportionality principle between variables that can be used as independent ones in the design and operation of a facility and variables representing the result of a given interaction mechanism, which can be considered as dependent variables. Such a principle does not involve linearity between said variables, because heat transfer is not so simple. A very good example of thermal coherence can be elaborated from next figures, taken from [20].

Figure 6 and Figure 7 show the energy and exergy efficiencies and the surface temperature of the collector pipe, for two different cases, which only differ in the temperature of the heat transfer fluid that absorbs heat while running inside the pipe. It is $400^{\circ} \mathrm{C}$ in the first case, and $300^{\circ} \mathrm{C}$ in the second one. They correspond to a certain design of a parabolic trough collector, but our interest is not concentrated on the exact numbers of each case, but in the general form characterizing this effect: a threshold is clearly seen in each case, both for the energy efficiency and the exergy efficiency. There is a large difference between them, for the same case, because the exergy efficiency takes into account that the source of the heat comes from the Sun, which radiates at a very high surface temperature $(5760 \mathrm{~K})$. It is obvious that our applications will be much colder than that. Anyway, it is 


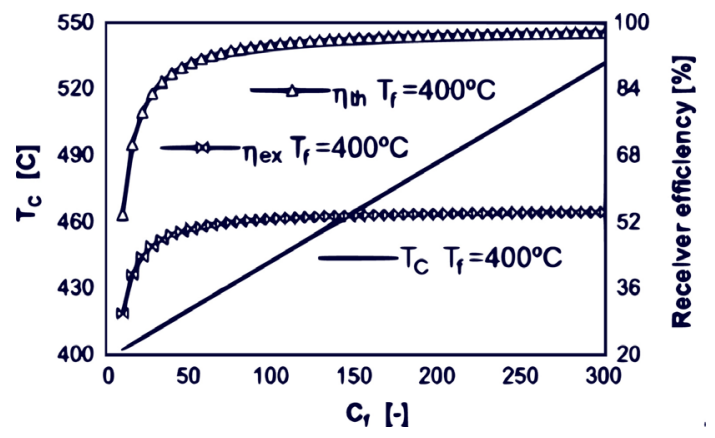

Figure 6. Collector efficiency and absorbing surface temperature vs. concentration factor, for a value of the temperature of the fluid $T_{f}=400^{\circ} \mathrm{C}$.

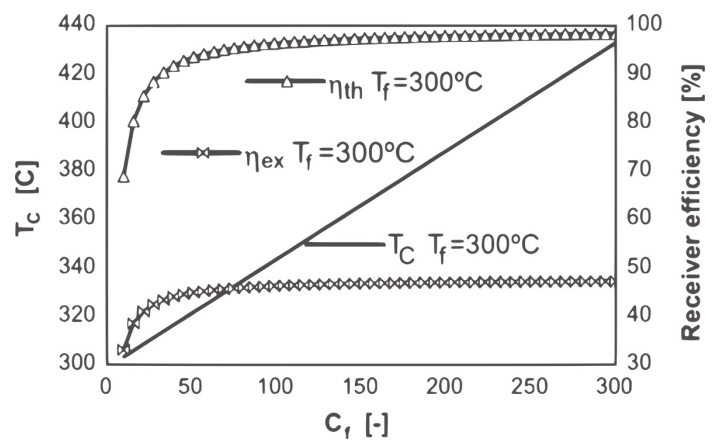

Figure 7. Collector efficiency and absorbing surface temperature vs. concentration factor, for a value $T_{f}=300^{\circ} \mathrm{C}$.

worth pointing out that efficiency increases very fast as the concentration factor increases, but a saturation level is reached at a given factor, which is higher for higher operating temperatures. In this case, a concentration factor 35 already gives an energy efficiency of $90 \%$ when the internal heat transfer fluid is $300^{\circ} \mathrm{C}$ (and the temperature of the tube is simply $310^{\circ} \mathrm{C}$ ). If the concentration factor is increased up to 300 , the efficiency rises to $97 \%$ but the temperature of the tube goes up to $410^{\circ} \mathrm{C}$. This is a simple zero-dimensional model with a balance for a given set of coating properties in the tube. The existence of such step in the efficiency curve is what matters. In addition, if the fluid is hotter, for instance, $400^{\circ} \mathrm{C}$, a $90 \%$ efficiency is achieved at a higher concentration value, close to 50 . In this case, if concentration is increased up to 300 , the efficiency also rises to $97 \%$ but the temperature jumps more, up to $530^{\circ} \mathrm{C}$.

Figure 8 taken from [21] depicts another important feature of solar radiation concentration onto the receiver. It is specific for a given Fresnel embodiment, Fresdemo (Almería Solar Platform, Spain) but the qualitative shape of the concentration factor iso-contours is utterly general. It is seen that the surface of the receiver with a factor higher than a selected threshold, decreases as the threshold increases. This fact represents a decreasing efficiency in the concentration process, as a bigger concentration factor is demanded. This effect can also be measured as the width of the stripe with concentration factors above a given value. For instance, in this case, a factor higher than 10 is attained in a $24 \mathrm{~cm}$ band, while a factor higher than 6 is only attained in a stripe $6 \mathrm{~cm}$ wide. 


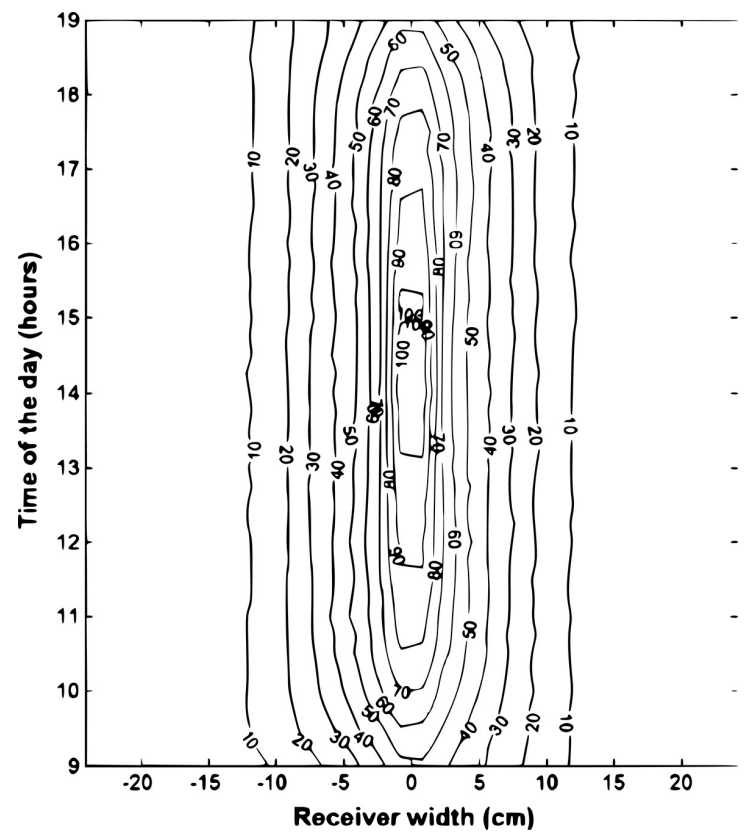

Figure 8. Radiation intensity $\left(\mathrm{kW} / \mathrm{m}^{2}\right)$ variation across the Fresdemo receiver width.

A coherent principle can be drawn from these pictures (and can be justified by equations and coefficients): once the operating temperature has been selected, it has no practical merit to use a concentration factor much higher than the threshold value. Note that the temperature of the tube increases quite a lot, and this means more thermal damage, and the need to use more expensive material. It must be taken into account that metals suffer from high temperatures because the elastic range becomes shorter as the temperature increases. So, to avoid plastic deformation in the tubes, either the temperature is kept at a reasonable value or better metals are chosen to that purpose.

An example of the complete challenge that exists inside this quest is Wind Energy technology. When Renewable Energies became an important research subject, in the mid 70s of last Century, as a reaction to the first Oil Crisis, there were many proposals for building modern windmills (aero-generators, as they are called modernly) and there was not a clear evidence of the best machine (in terms of efficiency and price). There were devices with vertical axis, and other with horizontal axis. The number of blades in a propeller was also an optimization unknown. The same can be said about the shape of the blades; some proposals followed the shape of helicopters (rectangular shapes) while others were after the classic style of "Spitfire". After 15 years of research, the 3-blade propeller was clearly dominant, and the machine was deployed in many countries. In Spain, the deployment started in 1990, with $1 \mathrm{MW}$ of electric windpower (over a total installed capacity of $42 \mathrm{GW}$; it went up to $1.9 \mathrm{GW}$ (over 51) in year 2000; and to $19.5 \mathrm{GW}$ (over 96) in 2010; and has reached $26 \mathrm{GW}$ (over 105) in 2020.

In solar thermal electric capacity, the deployment started much later, with 11 MW in 2006, for jumping to 500 in 2010 and 2 GW in 2012 and remains so. The 
strong deployment of those years was caused by the feed-in tariffs. It was not because of a technical success. However, windpower presented both effects, the political policy of fostering renewable energy sources and the technical success of a very good learning curve at international level. Capital cost went down to $1.2 \$ / \mathrm{W}$ in the early years of this Century, for large units (over $1 \mathrm{MW}$ ).

The solar thermal case is not so far a history of success, for several reasons. From them, two explanations are chosen, for designing a more adequate framework for the optimization of CSP:

- First, CSP has a lot of alternatives, and extensive research and technology projects are needed, in order to qualify properly the features of each line or alternative. It has been impossible to elaborate an international effort aimed at this characterization, and many potential solutions have been ignored by lack of time, lack of research manpower and lack of money.

- Second, CSP only works with direct solar radiation. This means that looking for a good site is mandatory. However, solar data are boundary conditions in our case, and cannot be modified by engineering practices. Nevertheless, there is the obligation to look for good sites for this objective.

\section{Application Example: Sundial}

The optimization of the system makes it necessary to reduce the cost function "C". Within this chapter the solar field (mirrors) acquires a special importance since a significant part of the total cost is due to it.

To reduce the investment costs of solar thermal collectors, a new Fresnel array prototype has been designed and built. The design tries not to compromise its efficiency in capturing solar radiation at high temperatures.

Figure 9 shows the decision tree that was followed to optimize the project by integrating technologies in a coherent way. Although the decision was essentially qualitative, it can be considered as a first step towards a specific project where numerical comparisons could be done on the basis of budgets for different lines of action. In the first steps of a development as this one, the system is still too open, and decision must be guided by expert judgement.

In Figure 9, technical coherence is established upon the concept of Moderate Domain, defined for this task. Note that the device is intended for its use as a tool for distributed generation, which means that pressure and temperature cannot pose serious safety problem. Coherently, the concentration factor should also be moderate. Otherwise, an extra cost would appear as a consequence of achieving in some part of the project a level of quality that is not necessary for its foreseen performance.

\section{Optimal Coherence between Concentrator and Receiver}

Formerly, a two-part model of thermal systems was analyzed for better understanding how to deal with a complex system, made of different types of technical complexity, because several fields of engineering knowledge should be embodied 


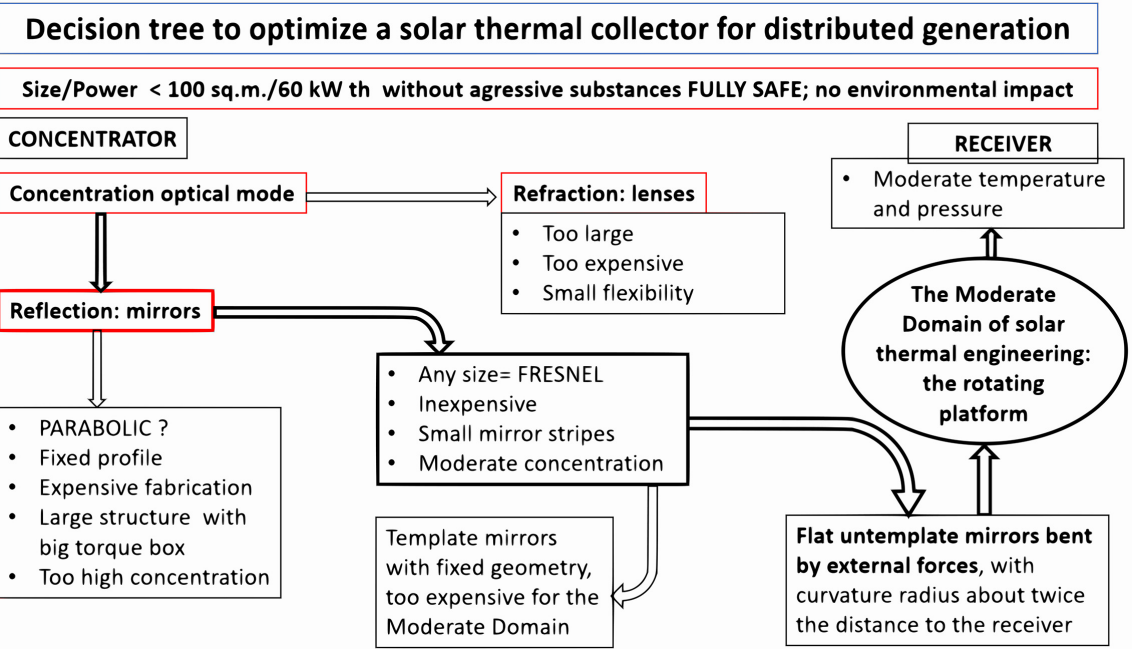

Figure 9. Decision tree followed to optimization

in ideal solution coming out from the optimization analysis. In previous section. Section 3.3 showed the algorithms underpinning the optimization procedure, being applied to the two main parts of the complete power plant: The Solar Field and the Block of Power.

In this section, the same methodology is applied to two sub-parts of a subsystem, and these parts are the Concentrator and the Receiver.

One of the main criteria for guiding this procedure is to select the value of each threshold just a little above the threshold itself, so starting the definition of what was called in the project the Moderate Domain, already introduced in Figure 9.

It is worth pointing out that coefficient $f$ stands for the efficiency in concentrating the solar radiation, above the required level; and coefficient $g$ is the efficiency of capturing the solar radiation (concentrated) by the heat transfer fluid.

Indeed, as will be seen in next pages, the objective of this first stage of this work line, was to characterize the concentrator, made of some singular innovations, intended to obtain good thermal performance with cheap or very cheap elements.

In [22]) the experimental facility of the Sundial concept was introduced.

This new configuration (Figure 10) is based on circular cylindrical mirrors, which can easily be made from planar ordinary mirror by applying a patented bending technique [23] that it is also very simple and cheap (Figure 11).

The development of the described patented technique is the key to reducing the costs of the system.

Something particularly difficult in concentrators is to keep the mirror well focused on the receiver when the mirrors rotate around their axis. It is difficult to achieve an accuracy greater than $100 \mathrm{mrad}$ on wide mirrors. All this translates into poor thermal performance.

To improve performance and solve the problem described, it is necessary to develop the patent described in [24]. 


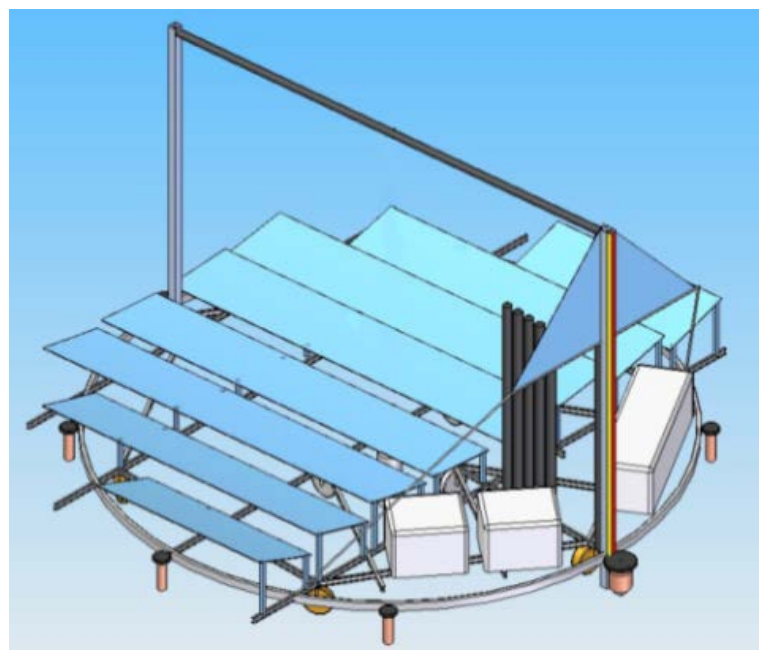

Figure 10. First proposal 3d representation.

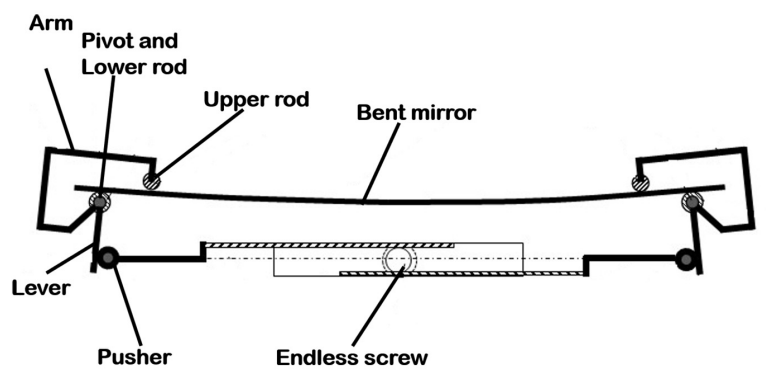

Figure 11. Cross-section of a bent mirror by torques applied to both lateral sides.

The development and application of these two patents has allowed us to build a prototype, and although the prototype is small ( $40 \mathrm{~m}^{2}$ of mirrors), as shown in Figure 1, it embodies all the pieces needed for an industrial device, and it can be considered as a reference for estimating costs in full scale system.

The main features of the prototype are:

- Horizontal platform rotating around a vertical axis

- Linear bent mirrors fixed to the platform reflecting the solar radiation into an absorber

- The platform rotates to allocate the Sun in the symmetry plane (Figure 12).

During the design and development phase we only look at the physical/theoretical aspects and, therefore, the results that would be obtained would fall within an ideal plane where the entropy would remain constant, but we know as the fundamental law of Nature that in all natural processes entropy grows. In the case of go from scientific knowledge and theoretical development to the technical level, manufacturing, there are numerous aspects that cause the increase of entropy. The higher the entropy, the less chance there is that the system will return to the desired state, tending towards chaos or an irreversible state, which would mean that the invention did not work, so the coherent integration of technology plays a fundamental role in said transition. 


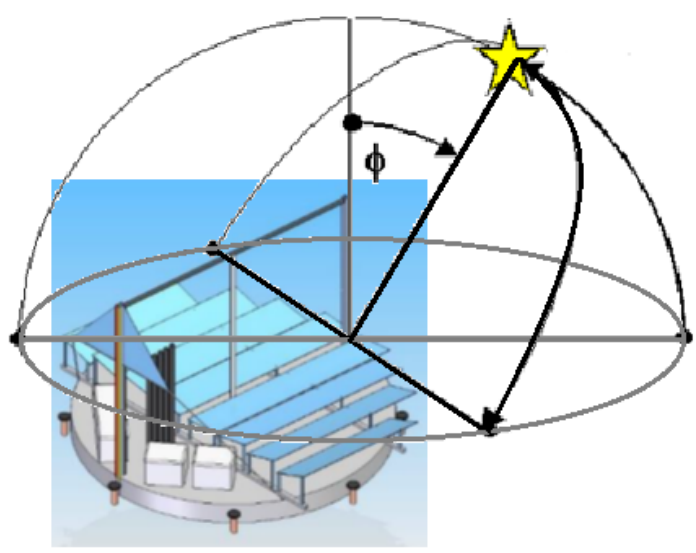

Figure 12. Sundial relative position from sun.

As already indicated in the scheme shown in Figure 4, the construction of the Sundial prototype requires the coherent integration of numerous disciplines and technologies to obtain the thermal performance calculated during the design phase, which is accomplished if we are able to limit the increase in system entropy.

The work is divided into the following parts or sections:

- Civil work and installation. Construction of the concrete slab and water and electric connection. The civil work includes the construction works of the concrete slab, placement of manholes, water pipes and electrical connection.

- Platform. Rotating platform base assembly. Detailed description of the construction and assembly of the support structure of the project.

- Rotation axis.

- Wheel selection and integration. Description of the wheel selection and its adaptation and assembly in the platform

- Motor-gearbox group for platform rotation. Calculations

- Electronic motor speed controller. Selection and configuration of speed controller.

- Solar filed layout. Mirror field.

- Mirror support structure

- Mirror bending system

- Mirror field adjustment procedure

- Receiver

- Receiver tubes assembly with two finishes, Natural nickel black, and polish nickel black.

- Heat exchanger

- Platform control and monitoring system

- Operating system based on real time: preempt Linux.

- Platform movement control system.

- Solar tracker system.

- Monitoring and data recording system, (type K thermocouple, wind speed, pressure, ...) 
We can see that there are a lot of technical and scientific fields in which such integration must be achieved, from civil engineering, through Industrial engineering, mechanical engineering, until electronics for the design of the control system, data acquisition and control, computer science for programming of the system.

The first point you want to achieve in an industrial design is to reduce costs so that the process is economically profitable, resources are limited.

All the mentioned points contribute to the increase its entropy and, therefore, to destabilize the system that will tend to disorder or chaos.

Thus, for example, a poor concrete base for which great flatness is required will make it impossible for the solar thermal plant to take advantage of the full range of available solar hours.

A slight inclination of the concrete slab results in the impossibility of achieving that all the reflected rays hit the receiver.

This effect can be observed in the Figure 13 and Figure 14 where a flat base makes the incident rays on the mirrors reach the receiver in a concentrated way. Figure 13 shows the ideal straight mirror section and solar beam reflection.

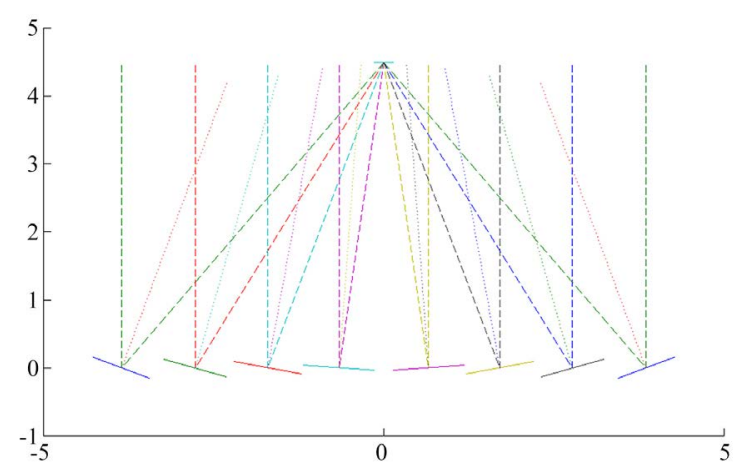

Figure 13. Straight section of mirrors.

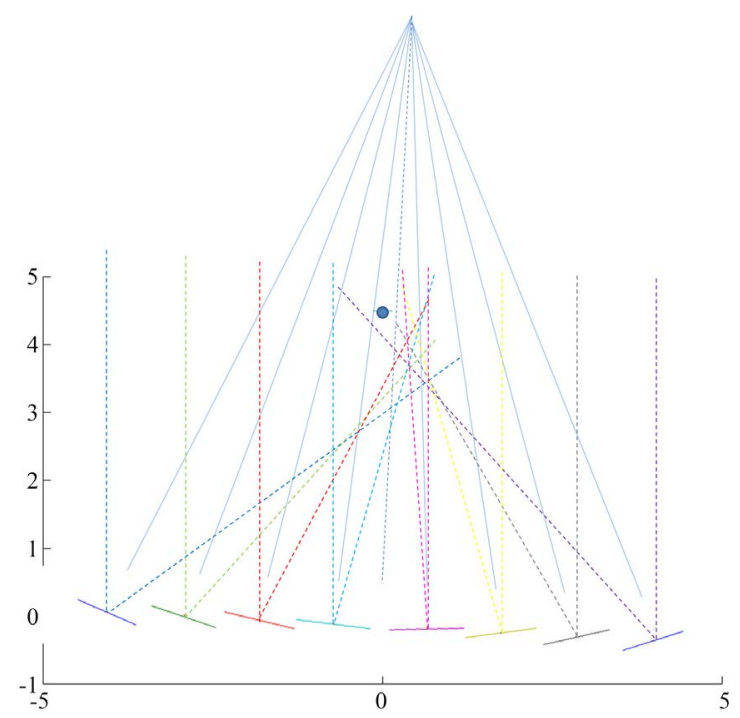

Figure $14.3^{\circ}$ Slab inclination effect. 
Figure 14 show the effect of a $3^{\circ}$ tilt slab of concrete. We can observe that no one sun ray touch the receiver, even with small errors, on the order of a tenth of a degree $\left(0.1^{\circ}\right)$ we can see this effect as shown in the Figure 15, on the right side of the image there are a calculated concentrated beam but due to the $0.1^{\circ}$ tilt on the concrete the real solar beam will be the left side of the image. This example has been calculated with the real data of the prototype with a receiver at $4.5 \mathrm{~m}$ high, and we can see that in the expected position of the receiver, the rays diverge. Figure 16 show horizontal tilt for every line of mirrors and relative position.

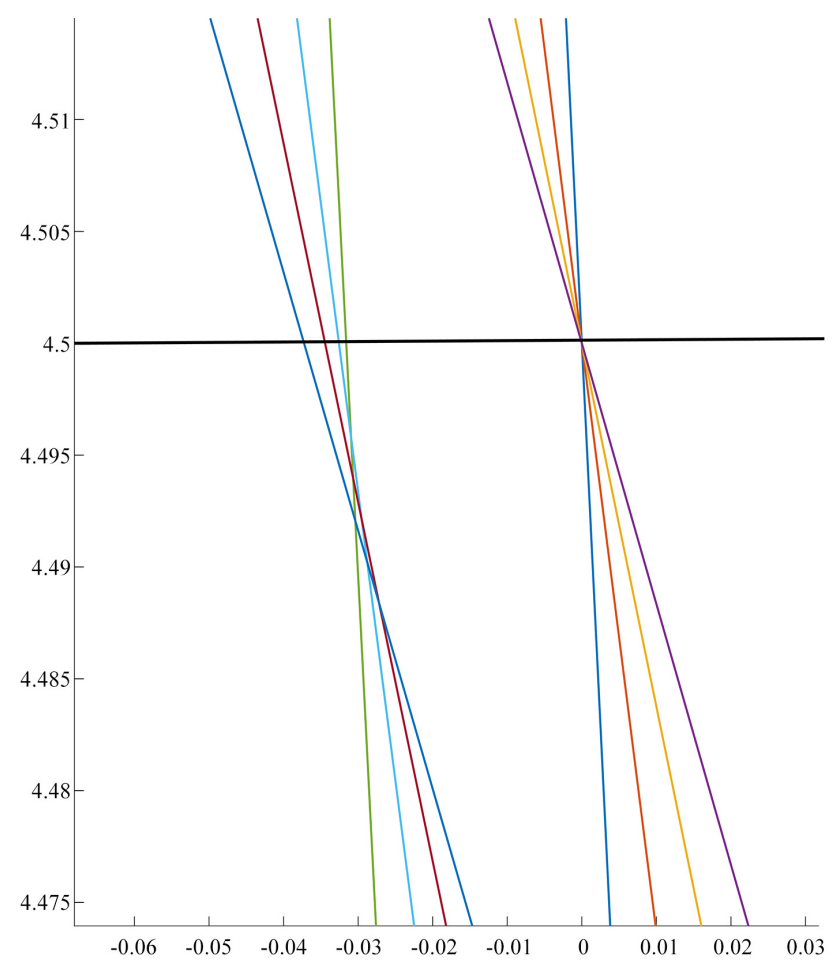

Figure 15. Effect over sunray at receiver level of $0.1^{\circ}$ flat concrete error.

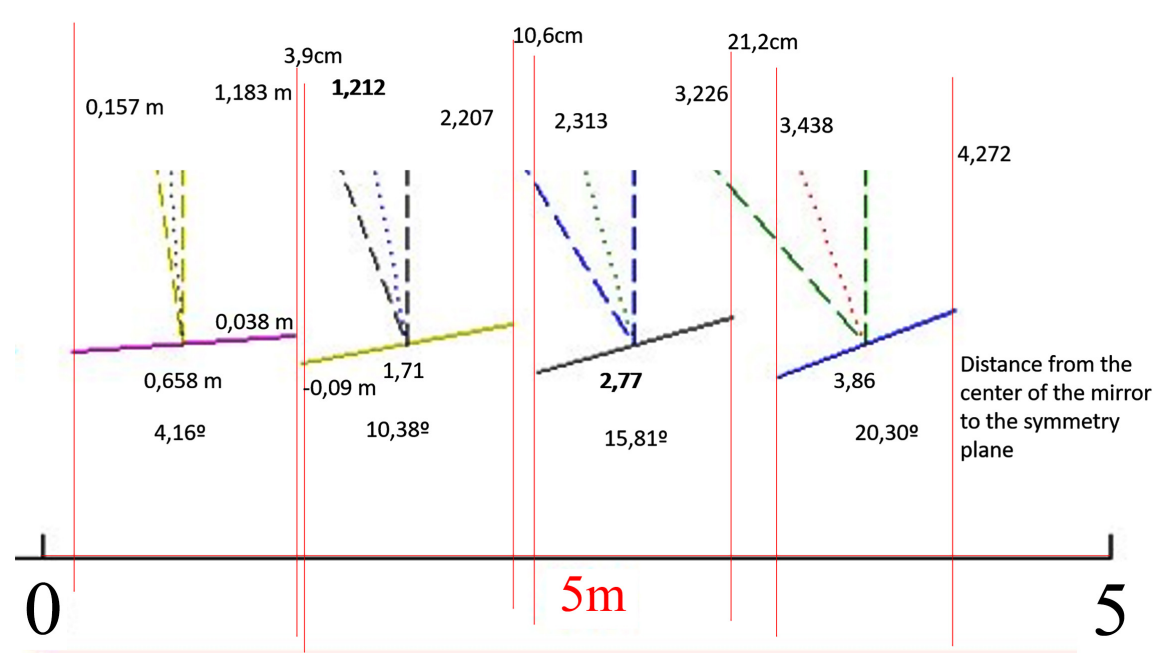

Figure 16. Relative mirror position in meter. 
In the same way, we are going to find many difficulties in achieving a robust system whose behavior approximates to the designed.

In design phase, calculations are made of the relative position of the mirrors, the curvature they must have, the distance at which the receiver must be located, the torque that be applied to the mirrors by the bending system. The position that each mirror must occupy is considered to avoid interferences and shadows between them.

As can be seen, the difficulty of the development, optimization and construction of a system as complex as the "Sundial" makes it necessary to apply a procedure such as that described in previous sections, a methodology for optimization of thermal systems.

The economic thermal optimization leads us to seek, on the one hand, the highest possible performance of the system, reducing the investment cost.

To get the best performance in the integration, we must consider what variables cause the increase in entropy of our system in order to be able to properly integrate by actuating to minimize or reduce these errors on the final system.

The set of variables we can study can be divided into two groups or categories: the first group represent the design parameters of the system. The initial design of the system is carried out considering the ideal conditions and defines its initial state or state of minimum entropy.

The second group of variables are those that really affect entropy increase, these variables should be independent variables between them, but this is not always easy. We can associate each part or section described with one or more variables. Thus, we would have defined the integration based on a series of variables related to the previous described parts:

- Civil work and installation.

- Flatness of concrete

- Platform.

- platform base assembly.

- Wheel selection and integration.

- Motor-gearbox

- Electronic motor speed controller.

- Solar filed layout. Mirror field.

- Mirror support structure

- Mirror bending system

- Mirror field adjustment procedure

- Receiver

- Heat exchanger

- Platform control and monitoring system

- Operating system based on real time

- Platform movement control system.

- Solar tracker system

- Monitoring and data recording system 
The main proposed variables are:

- Flatness of concrete.

- Mirror position optimization.

- Accuracy in the mirror bending system.

- Mirror support structure building.

- Assembly precision.

- Solar tracking and platform movement.

- Maintenance. Cleaning the mirrors, dust. A serious inconvenience.

For each variable, the cost benefit ratio must be analyzed to allow us to obtain the highest ratio between useful energy and investment cost as, as stated in Equation (10).

\section{Sundial Prototype}

A small-scale prototype has been built in Tecnogetafe campus of UPM [25], with a very reduced budget and a nominal power over $30 \mathrm{~kW}$ with a final temperature above $300^{\circ} \mathrm{C}$, with near $40 \mathrm{~m}^{2}$ of mirrors in 8 stripes.

SUNDIAL has been developed by the Technical University of Madrid research group GIT (Grupo de Investigaciones Termoenergéticas) in collaboration with Fundación para el Fomento de la Innovación Industrial, F2I2 [26] with own founds of both institutions. The main features of the prototype are summarized on Table 1.

Figure 17 shows a general scheme of the prototype.

The materialization of these developments was show in Figure 1, where it can be seen the complete installation of the mirros with their clips in the platform and the illuminated receiver by the concentrated radiation.

The place chosen for the construction of the prototype is the South Technological Area, Tecnogetafe (Figure 18), Science and Technology Park of UPM [25].

Tecnogetafe, is located in the Madrid municipality of Getafe where UPM has enough space and capacity to develop the prototype.

Table 1. Sundial prototype main features.

\begin{tabular}{cc}
\hline Description & Value \\
\hline Thermal power & $30 \mathrm{~kW}$ \\
Basic diameter & $8 \mathrm{~m}$ \\
Aperture area & $20.25 \mathrm{~m}^{2}$ \\
Receiver diameter & $140 \mathrm{~mm}$ \\
Working fluid & Air \\
Working pressure & Atmospheric pressure \\
Working temperature & Up to $325^{\circ} \mathrm{C}$ \\
Receiver absorptivity/emissivity & 80 \\
\hline
\end{tabular}




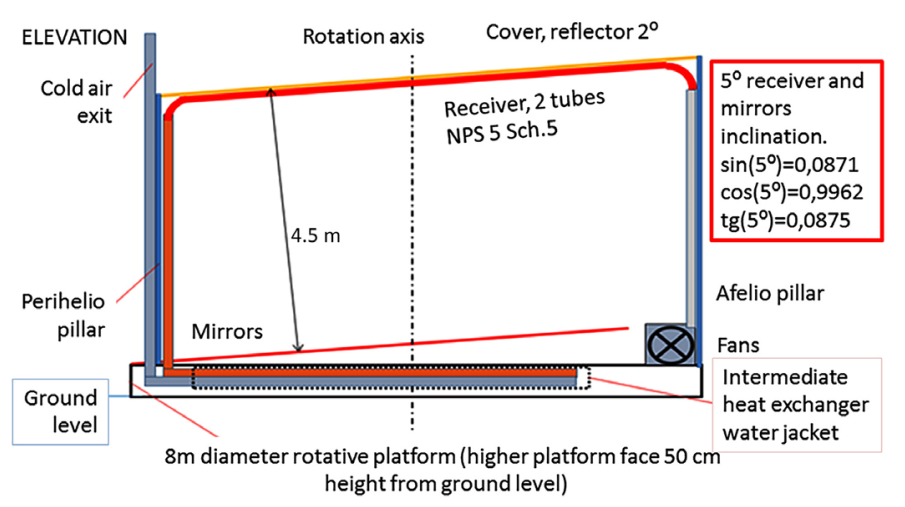

Figure 17. Elevation plan. General prototype scheme.

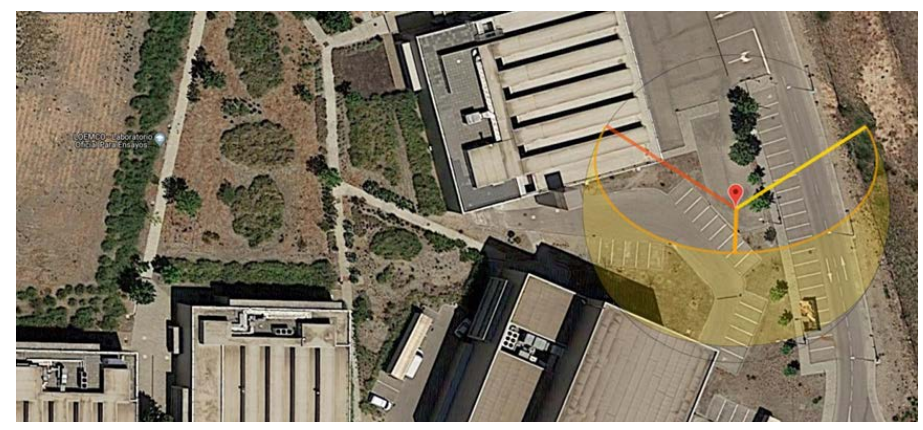

Figure 18. Prototype location.

\subsection{Civil Work and Installations}

The first variable is flatness of concrete (Figure 19). Considering the optimization procedure that has been presented in previous sections, this variable is part of what we have called cost function " $C$ ".

At this point it is important to focus on a correct drafting of the specification that is delivered to the builder and on the monitoring of the process. The way to reduce costs is through the negotiation and search for offers to do such work.

\subsection{Platform}

The base of the platform has been conceived as a meccano model construction system. This solution allows us to assemble the platform ourselves without the need assistants.

We use $3 \mathrm{~mm}$ thickness rectangular profiles of $60 \times 40 \mathrm{~mm}$ (Figure 20 and Figure 21)

The basic configuration is a radial configuration and can be seen in the Figure 22.

In the perihelion, we mount the air outlet and connection pipes between the receiver and the heat exchanger.

\subsection{Solar Field Layout}

The second group of variables that we evaluate are the related variables to the solar field layout. 


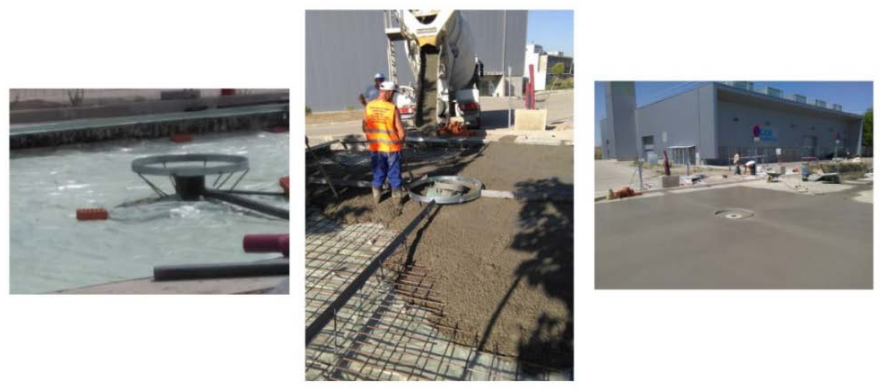

Figure 19. Concrete Slab construction images.
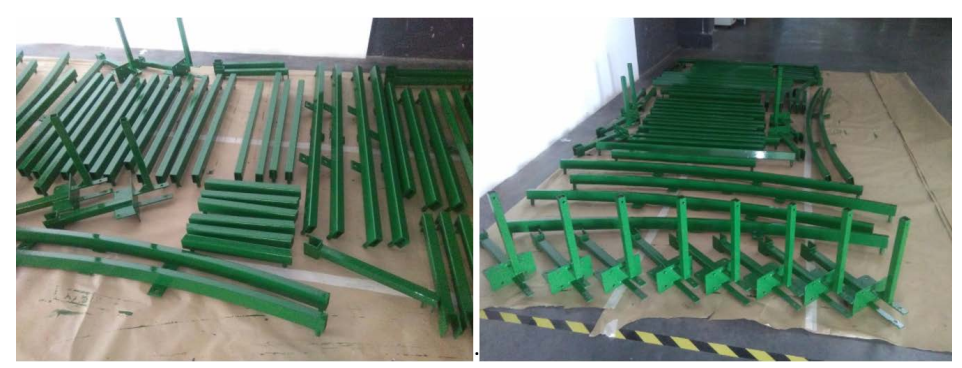

Figure 20. Platform parts before mounting.
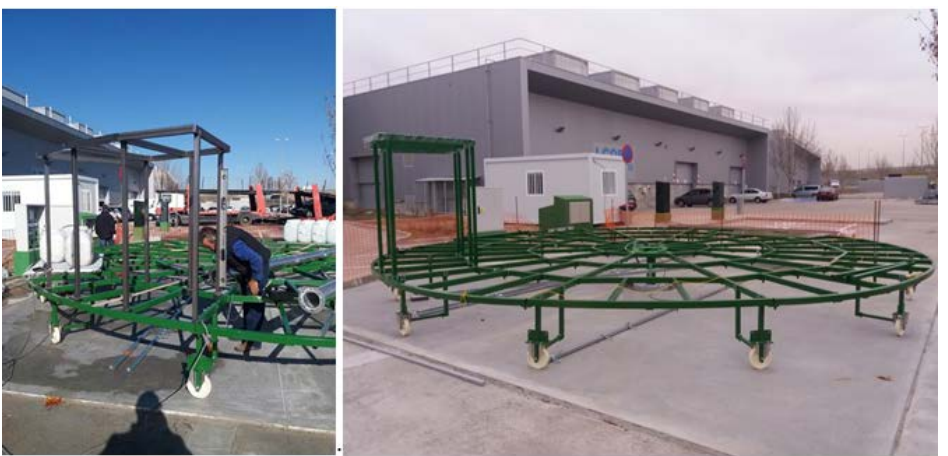

Figure 21. Complete view base mounted.

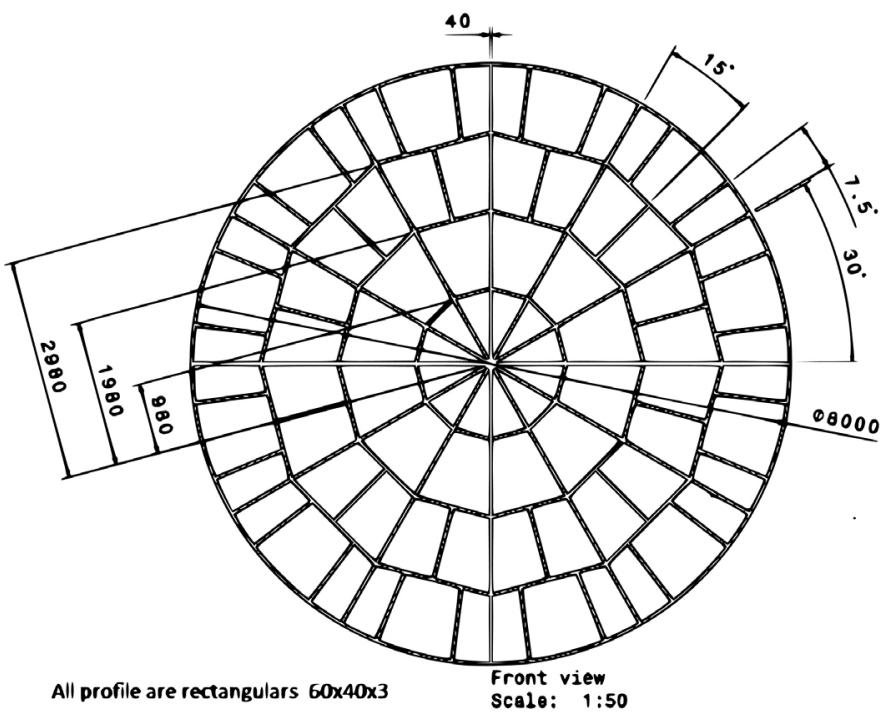

Figure 22. Base plan. 
As baseline information, we will use as reference system the coordinate system show in Figure 23, where the perihelion is located at the coordinate $(0,0,0)$ and $\mathrm{Y}$ axis inclination Figure 24.

The optimization of the position of the mirrors, this variable is linked to a design one. As explained before, during the design phase the initial conditions for the construction of the complete system are established, however when executing it, elements may arise that interfere with the location of the pillars and forces a rethinking and modification of the system.

For example, in the case of the mirror field, and specifically the location of the supports of the central mirrors, it has been necessary to move their location and recalculate the supports due to mechanical interference with the receivers.

The design data we have are the relative positions of the mirrors, their dimensions and tilt angles for each group of mirrors.

Transferred this information to three dimensions we have the $3 \mathrm{~d}$ view (Figure 25, and Figure 26).

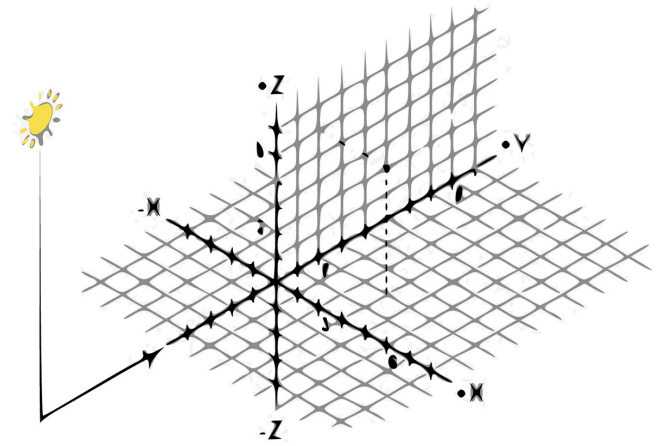

Figure 23. Reference system.

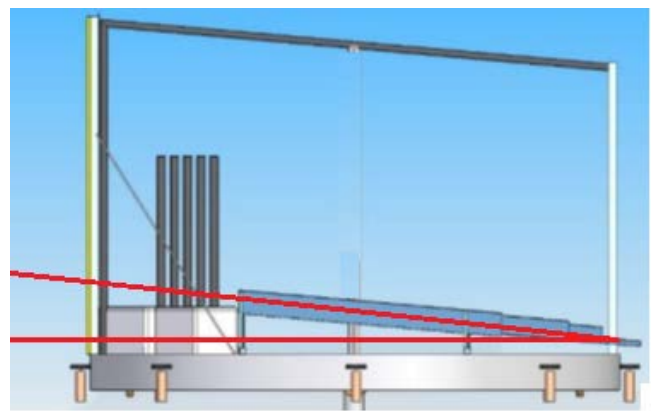

Figure 24. Mirrors Y axis angle inclination.

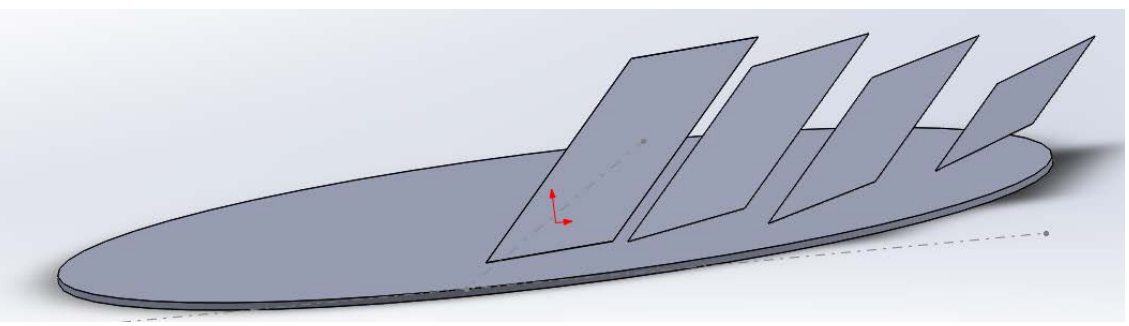

Figure 25. 3d view of half the mirror field. 


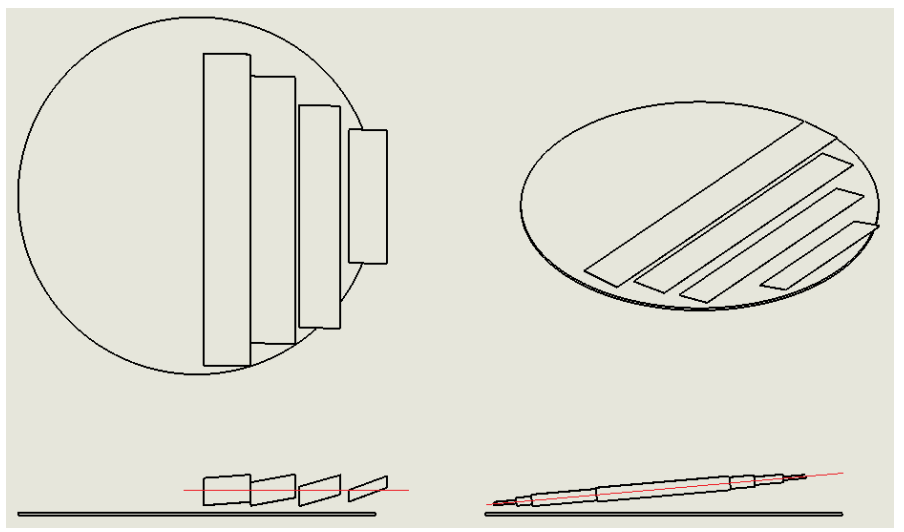

Figure 26. General view.

The $3 \mathrm{~d}$ view shown allows us to verify that the calculations are correct, the mirrors are aligned in their axes and the angle of inclination of each mirror corresponds to the initial data on both the $\mathrm{X}$ and $\mathrm{Y}$ axis.

At this point we must decide on the design and construction of the mirror support structure. The most immediate thing is to think of a steel structure versus using structural aluminum profiles. Although the price of steel is lower and therefore more attractive to optimize and reduce costs, this is not the case, especially in the construction of a prototype. Aluminum profiles give us greater freedom in correcting faults, require the use of simple, low-cost tools for assembly, and reduce assembly times.

\subsubsection{Mirror Support Structure}

Mirror support structure prototype is made of aluminum Bosch Rexroth $45 \times 45$ profile (Figure 27).

This type of structure is not the most economical and in a final construction it would be replaced. However, for a prototype like this, is the solution that gives us greater versatility and ease of work.

To guarantee a homogeneous movement of the mirror folding system, a ribbed round tube, Bosch Rexroth $28 \mathrm{~mm}$ round tube, has been chosen (Figure 28).

In a first approximation the structure would be formed by a series of pillars (Figure 29) however, since the local distributor provides us the profiles length pieces of $2 \mathrm{~m}$ and $3 \mathrm{~m}$ we have to divide every structure into two or three part as seen in the Figure 30.

The division of a large structure into smaller structures contributes to the modularity of the system and reduces its complexity. The scheme shown in Figure 30 is reflected in Figure 31 and Figure 32 where several modules or structures of mirror supports can be seen.

\subsubsection{Mirror Bending System. Model Design}

The system for bending the mirrors is the application of patent No. ES2596294 B2 from [23], the variant used is the referenced inside the patent document (Figure 11) "the variant of conformation of the clamp, with arm in C". 
The first element we need is the design of the clamp (Figure 33).

For the calculated sizes of the mirrors, we have in mind that the point of contact of the clamp that supports the mirror is $5 \mathrm{~cm}$ from the inside wall of it (providing at least $5 \mathrm{~mm}$ of safety margin) (Figure 34 ).

Figure 35 perfectly illustrates the operation of the system.

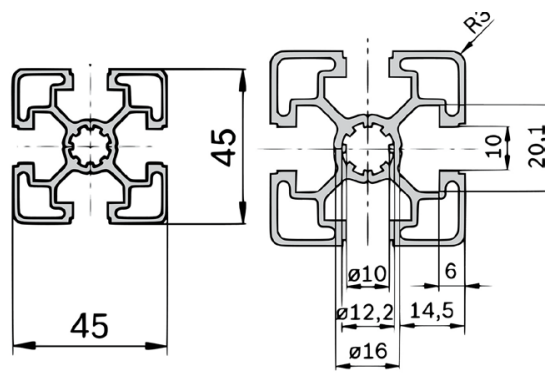

Figure 27. Aluminum profile.

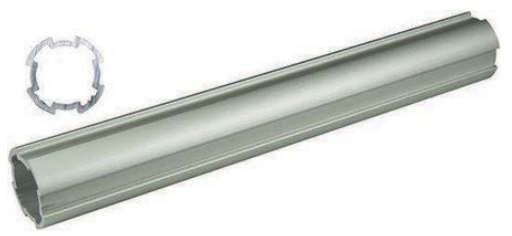

Figure 28. Round tube aluminum profile.

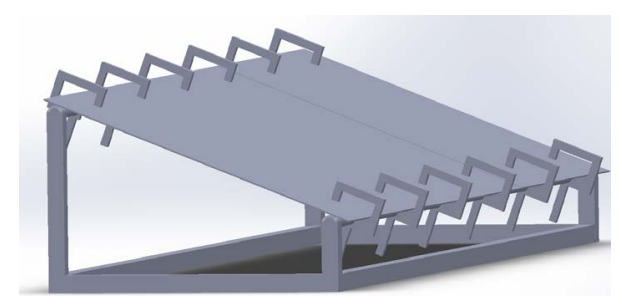

Figure 29. 3D mirror support structure view.

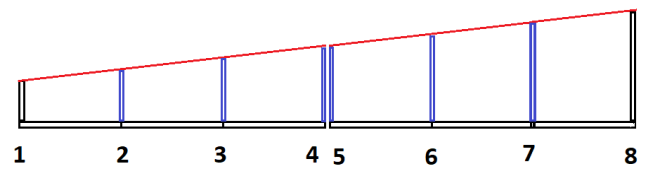

Figure 30. Division example.

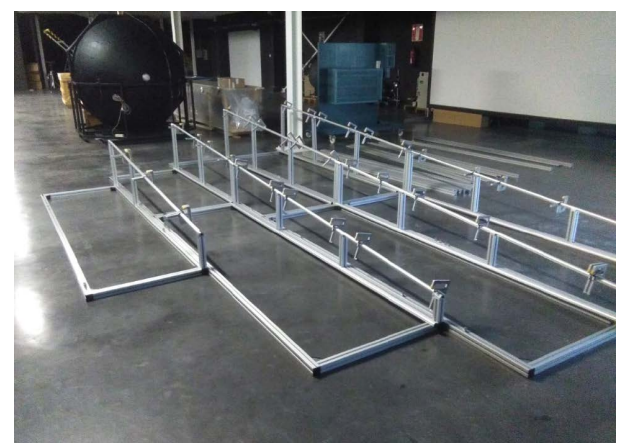

Figure 31. Assembly process. 


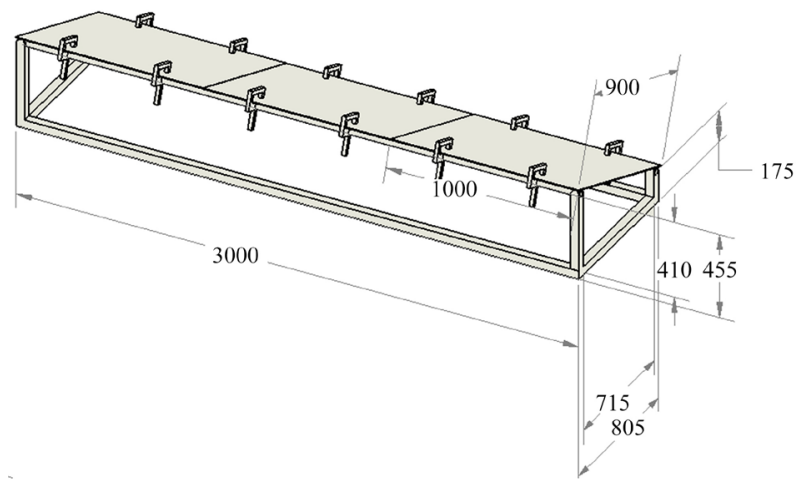

Figure 32. Dimension of an $3 \mathrm{~m}$ sample structure.
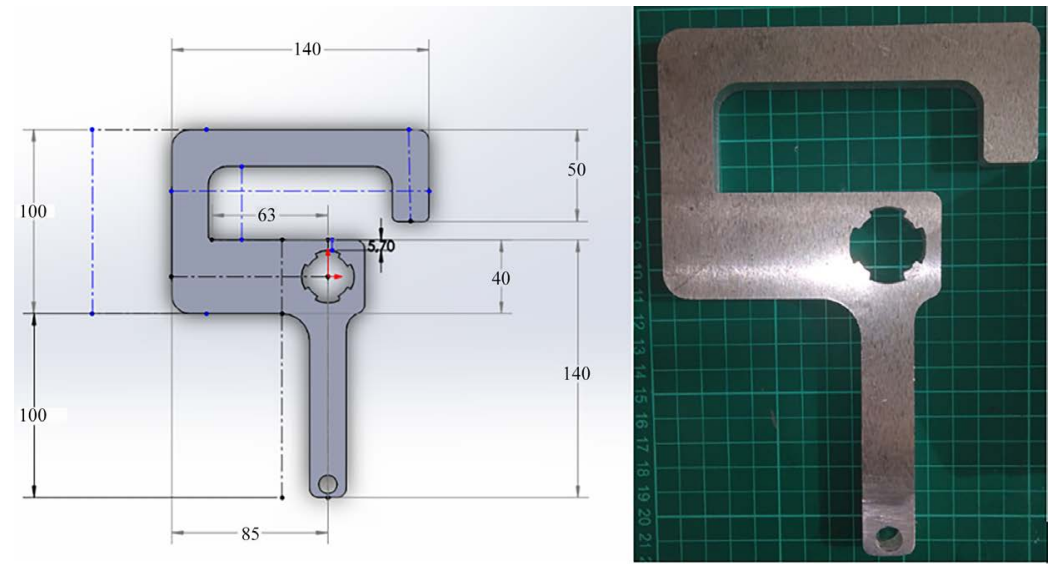

Figure 33. Final clamp model.

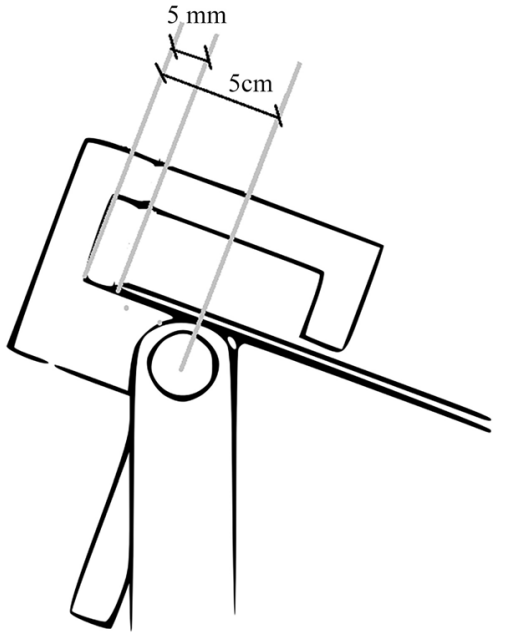

Figure 34. Safety distant from mirror to clamp.

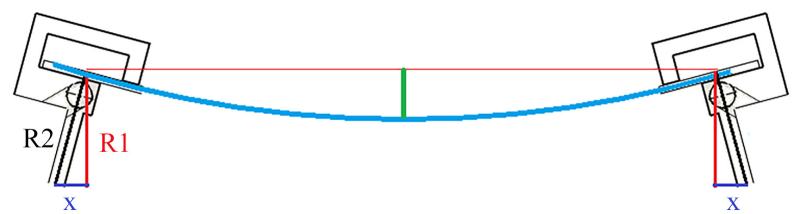

Figure 35. Illustrative image of bending system working. 


\section{Results and Discussion}

As a result of the application of the proposed method, a prototype has been built where the two initial assumptions or premises are clearly evidenced.

On the one hand, the designed apparatus fulfills its function in a remarkable way, managing to concentrate the solar radiation in the receiver and getting temperatures over $300^{\circ} \mathrm{C}$.

As an example, the images resulting from monitoring the temperatures in the receiver with thermocouples and thermographic images taken with a thermal camera are show in Figure 36 and Figure 37. Table 2 shows values for Figure 37 and these data are very close to those obtained by finite element simulation of the receiver, an example is show in the Figure 38.

Secondly, the prototype has been built with a cost per installed watt that is significantly lower than the traditional costs of a conventional solar thermal plant.

If we compare the real cost of our prototype with the real construction costs obtained from an engineering consulting company ([27] [28]), from this source we know that the main cost of thermal plant are Solar Field and Mechanical assembly. These two items represent more than $50 \%$ of the total cost.

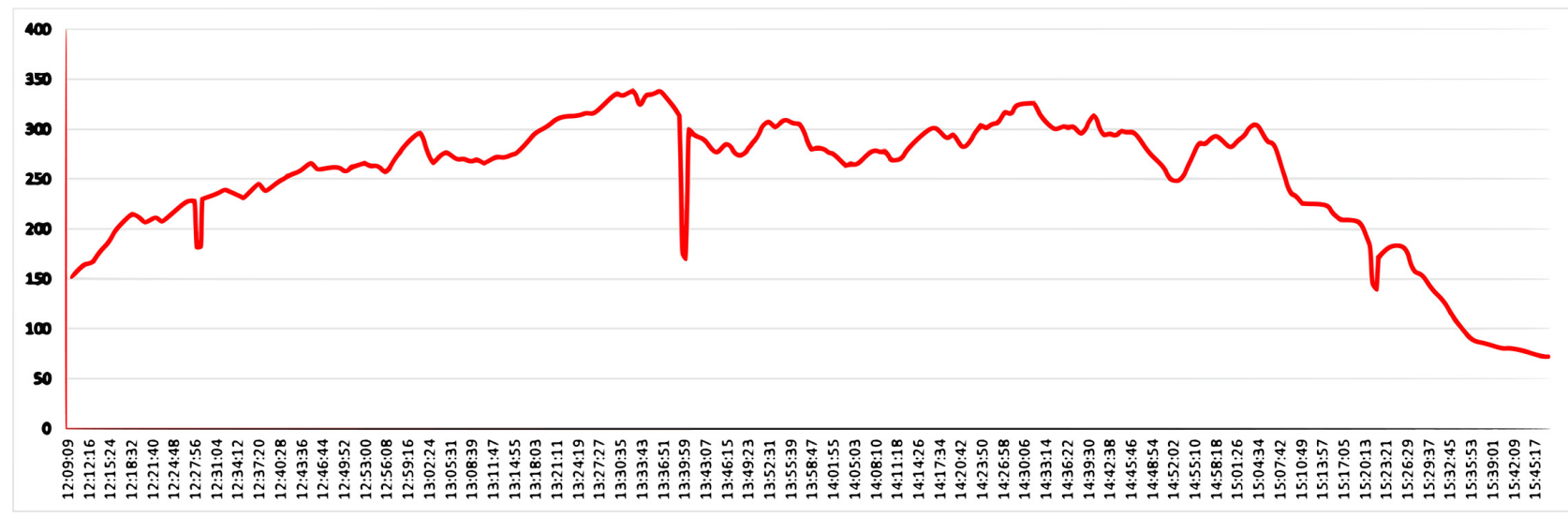

Figure 36. Surface temperature of the right pipe Thermocouple ${ }^{\circ} 2$. Aphelion.

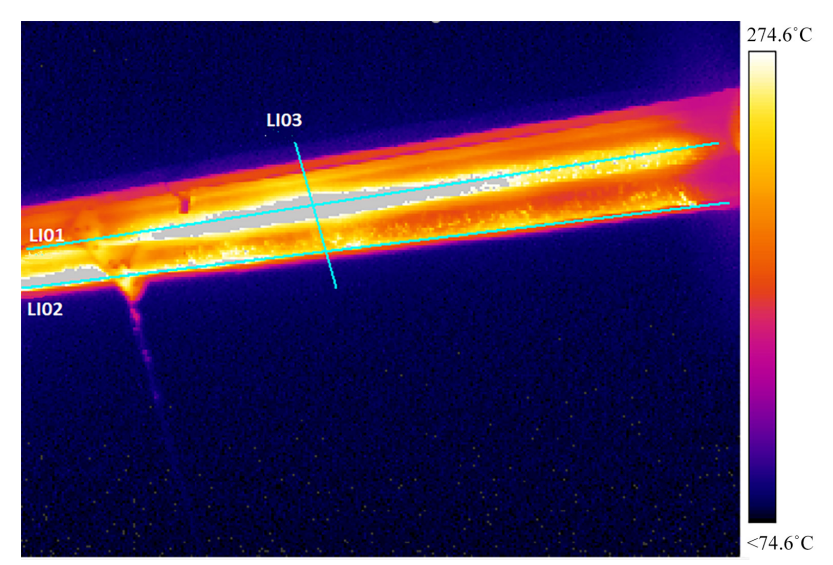

Figure 37. Receiver Thermal imagen from FIR 550/Agema 550 camera. Surface temperature. 


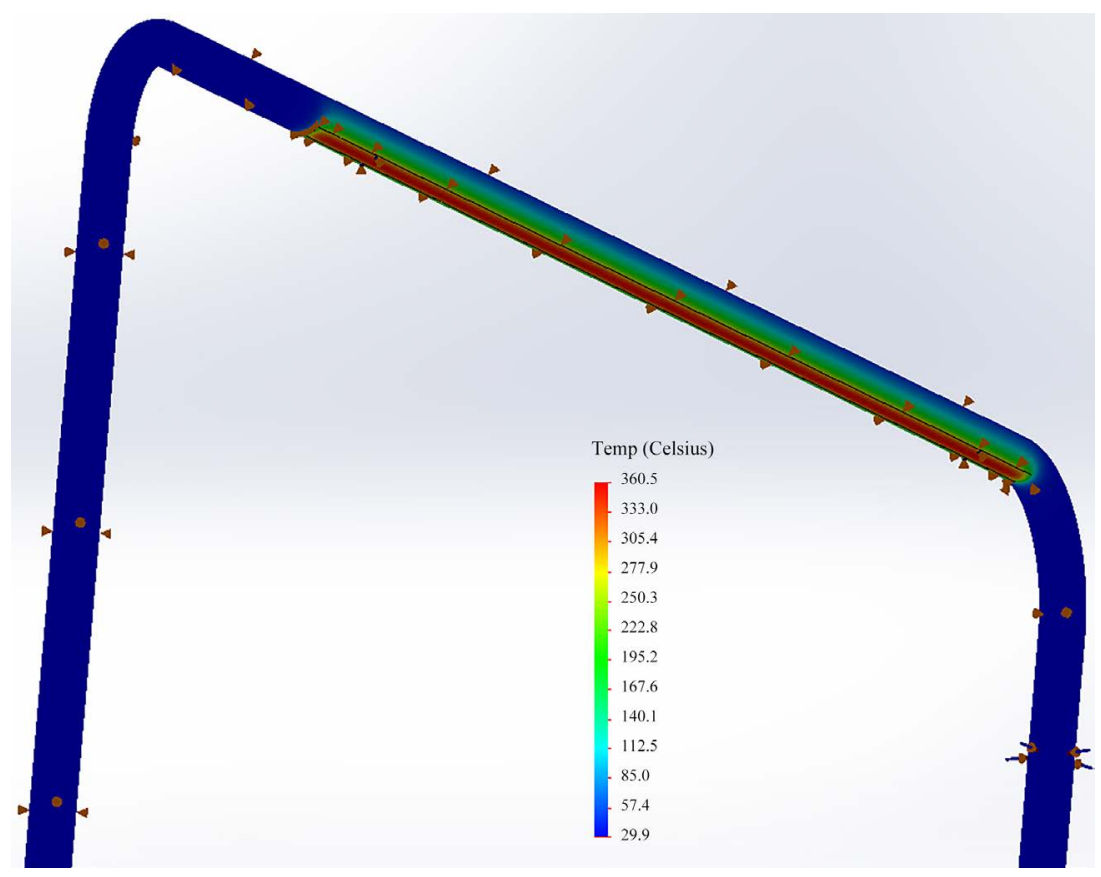

Figure 38. Simulation example. Receiver temperature.

Table 2. Temperature data for Figure 37.

\begin{tabular}{|c|c|c|c|c|c|c|}
\hline Label & Value $\left[{ }^{\circ} \mathrm{C}\right]$ & Min & Max & Min-Max & Avg & Stdev \\
\hline image & & $<76.4$ & $>337.1$ & 260.7 & & \\
\hline LI01 & & 184.2 & $>337.1$ & $>153.0$ & 269.8 & 28.9 \\
\hline LI02 & & 135.9 & 316.4 & 180.5 & 243 & 40.2 \\
\hline LI03 & & $<76.4$ & 331.2 & 254.8 & 192 & 88 \\
\hline
\end{tabular}

From this data we can see that the cost are:

- Solar field cost is $1.53 \$ / \mathrm{W}$ and

- mechanical assembly cost is 1.07 \$/W

As indicated in Table 1, our prototype has been designed with a power of 30 $\mathrm{kW}$, the total cost of solar field and support were $22.589 .00 \$$ that is, a cost of solar field of $0.76 \$ / \mathrm{W}$ and the mechanical assembly was $15.976 .00 \$$, that is 0.53 $\$ / \mathrm{W}$.

Even considering that horizontal RFC will collect around a $20 \%$ less energy per mirror area than a PTC [29], and correcting the cost of Sundial with this factor, Sundial has a better investment cost ratio. (see Table 3, Table 4 and Figure 39).

Under these conditions, the saving in solar field and construction costs are very high, up to $38 \%$ and moving this data to the total cost of the installation would mean a global saving of $20 \%$.

The costs of the construction of our prototype have been made through access to the local market through retailers, this means that in an industrial construction with economies of scale, significant cost reductions can be achieved. 


\section{Conclusions and Future Work}

The Sundial prototype (Figure 40) was a demonstration that a coherent integration of technologies is a way to reach practical optimization. The work on this line is far from finished because the prototype has not yet been connected with a suitable block of power. In the first steps of this project, emphasis was put in the solar field, which is the specific part of a solar thermal power plant.

Table 3. Cost data summary of solar field and mechanical assembly from 50 MW CSP in 2016.

\begin{tabular}{ccc}
\hline Description & Cost & \%Over total cost \\
\hline Solar field & $76.409 .214 .17 \$$ & $33.00 \%$ \\
Mechanical assembly & $53.354 .145 .30 \$$ & $23.04 \%$ \\
\hline
\end{tabular}

Table 4. Summary compared construction cost per Watt.

\begin{tabular}{cccc}
\hline Description & Reference CSP & Sundial & Sundial Normalized \\
\hline Solar field $[\$ / \mathrm{W}]$ & 1.53 & 0.76 & 0.95 \\
Mechanical assembly $[\$ / \mathrm{W}]$ & 1.07 & 0.53 & 0.67
\end{tabular}

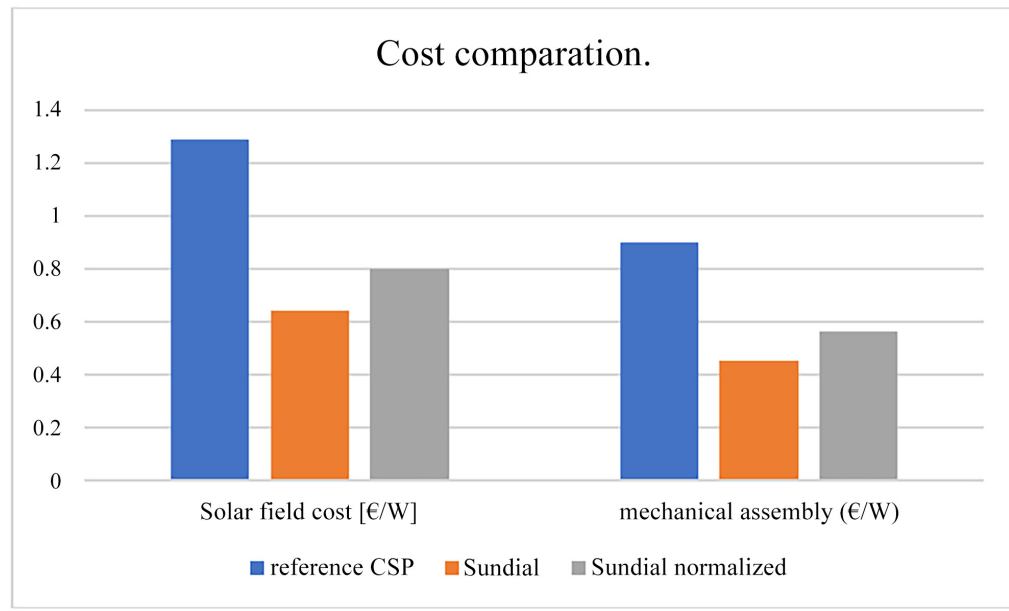

Figure 39. Comparation cost per Watt.
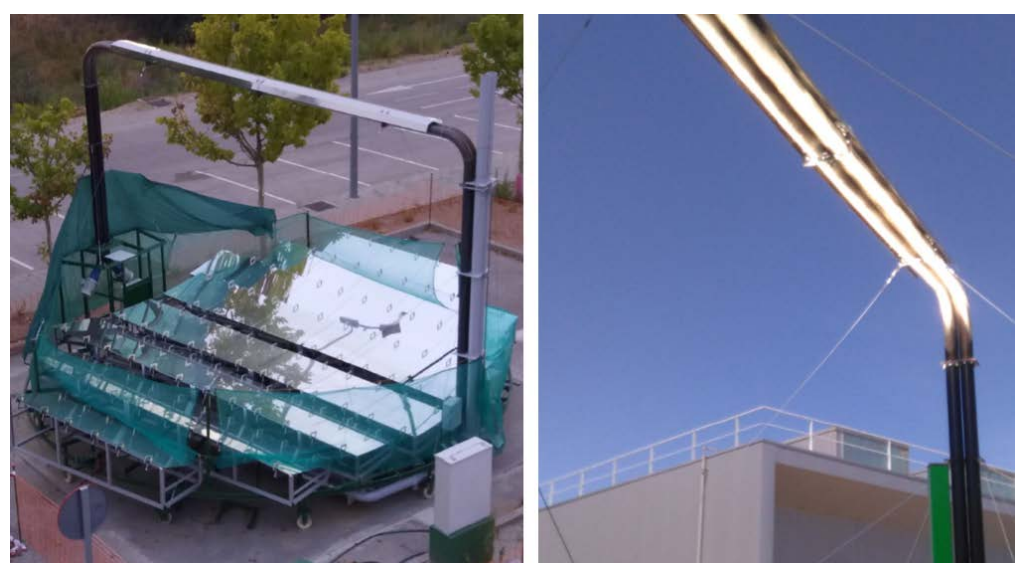

Figure 40. General view of Sundial prototype and received concentration view. 
Once the goal of the system to be developed was clearly defined as a tool for distributed generation, the concept of Moderate Domain was coined, and it led the project conceptual design to a novelty of concentrating mirror construction, using simple flat mirrors that were bent by applying external pairs of force on the borders parallel to the rotation axis.

Additionally, another novelty was proposed for making the tracking of the Sun in a simple and robust way: all elements of the collector are placed on a platform rotating around a vertical axis. The rotation speed equals the apparent speed of the Sun in the azimuthal plane of the platform. The geometric relations among the collector elements are kept constant along time. Of course, a flexible connection must be provided between the collector in the platform and the ground, A possibility is to use a Stirling engine plus a generator to produce electricity, which can easily be evacuated by a twisted cable. The alternative is to evacuate the heat carrier fluid through a flexible hose. The limit for a material with good properties for that purpose is about $300^{\circ} \mathrm{C}$ and $2 \mathrm{MPa}$, which is enough for moderate-efficiency applications.

Using $3 \mathrm{~d}$ design and simulation software, a first model of a simple receiver has been made. A simulation has been run on this model where it is obtained that the expected temperature in the receiver is close to $360^{\circ} \mathrm{C}$.

The application of the concept of Coherent Integration of Technologies has allowed us to obtain results very close to those expected and calculated through finite element simulations, these simulations have been contrasted with the capture of thermographic images.

The experience acquired in the construction of the first prototype of this kind, plus the continuation of analytical developments point out to the continuation of this research looking for improvements under the same technical spirit explained in this paper.

The construction of a second prototype with a degree of freedom for the mirror to rotate slightly around axes parallel to the receiver, is foreseen to start in the first half of 2021.

\section{Funding}

This paper has been partially supported by the project "ACES 2030 CM: Energía solar de concentración" granted by Comunidad de Madrid (Madrid regional government) and European Structural Funds. ACES2030-CM project (S2018/ EMT-4319).

\section{Acknowledgements}

Discussions with the rest of our Research Group are highly recognized.

Special thanks to "Fundación para el Fomento de la Innovación Industrial" for their valuable support.

The Sundial project was awarded the second prize of Innovatech 2019, a contest organized by UPM every year for stimulating innovation (about 100 ideas 
and initiatives participate in these contests, and the members of the jury are not from UPM).

\section{Conflicts of Interest}

The author declares no conflicts of interest regarding the publication of this paper.

\section{References}

[1] Monteith, J.L. (2013) In: Unsworth, M.H., Ed., Principles of Environmental Physics Plants, Animals, and the Atmosphere, 4th Edition, Academic Press, Oxford.

[2] Muradov, N.Z. and Veziroğlu, T.N. (2008) "Green" Path from Fossil-Based to Hydrogen Economy: An Overview of Carbon-Neutral Technologies. International Journal of Hydrogen Energy, 33, 6804-6839. https://doi.org/10.1016/j.ijhydene.2008.08.054

[3] Chiras, D.D. (2017) Power from the Sun: A Practical Guide to Solar Electricity (Revised Second Edition). New Society Publishers, Gabriola Island.

[4] Luque, A.L. and Viacheslav, A. (2007) Concentrator Photovoltaics. Springer, Berlin. https://doi.org/10.1007/978-3-540-68798-6

[5] Miyazaki, Y. (2015) Liouville's Theorem and Heat Kernels. Expositiones Mathematicae, 33, 101-104. https://doi.org/10.1016/j.exmath.2014.02.001

[6] Stoecker, W.F. (1989) Design of Thermal Systems. 3th Edition, McGraw-Hill, New York.

[7] Aseri, T.K., Sharma, C. and Kandpal, T.C. (2020) Estimating Capital Cost of Parabolic Trough Collector Based Concentrating Solar Power Plants for Financial Appraisal: Approaches and a Case Study for India. Renewable Energy, 156, 1117-1131. https://doi.org/10.1016/j.renene.2020.04.138

[8] Fundación de la Energía de la Comunidad, de Madrid and Madrid (Comunidad Autónoma) Consejería de Economía, y Hacienda (2012) Guía técnica de la energía solar termoeléctrica. Madrid Fundación de la Energía de la Comunidad de Madrid Consejería de Economía y Hacienda, Madrid.

[9] Jaluria, Y. (2008) Design and Optimization of Thermal Systems. 2nd Edition, CRC Press, Boca Ratón.

[10] Steihaug, T. (1983) The Conjugate Gradient Method and Trust Regions in Large Scale Optimization. SIAM Journal on Numerical Analysis, 20, 626-637. https://doi.org/10.1137/0720042

[11] Wang, Z. (2019) Design of Solar Thermal Power Plants. CIP: Academic Press, London. https://doi.org/10.1016/B978-0-12-815613-1.00003-1

[12] Tsatsaronis, G. (2007) Definitions and Nomenclature in Exergy Analysis and Exergoeconomics. Energy, 32, 249-253. https://doi.org/10.1016/j.energy.2006.07.002

[13] Bai, Y. and Bai, Q. (2010) Chapter 14 Heat Transfer and Thermal Insulation. Elsevier Inc., Amsterdam. https://doi.org/10.1016/B978-1-85617-689-7.10014-7

[14] Boukelia, T.E., Mecibah, M.S., Kumar, B.N. and Reddy, K.S. (2015) Investigation of Solar Parabolic Trough Power Plants with and without Integrated TES (Thermal Energy Storage) and FBS (Fuel Backup System) Using Thermic Oil and Solar Salt. Energy, 88, 292-303. https://doi.org/10.1016/j.energy.2015.05.038

[15] Rovira, A., Martínez-Val, J.M. and Valdés, M. (2016) Thermoeconomic Coherence: A Methodology for the Analysis and Optimisation of Thermal Systems. Entropy, 
18, 250. https://doi.org/10.3390/e18070250

[16] Castells, X.E. (2012) In: e-Libro, C., Ed., El modelo energé tico español. Ediciones Díaz de Santos, Madrid.

[17] Kotas, T.J. (1985) The Exergy Method of Thermal Plant Analysis. Butterworths, London, Boston.

[18] El-Sayed, Y. (2003) The Thermoeconomics of Energy Conversions. Elsevier, Amsterdam.

[19] International Renewable Energy Agency (IRENA) (2012) Concentrating Solar Power. International Renewable Energy Agency. https://www.irena.org/-/media/Files/IRENA/Agency/Publication/2012/RE Technol ogies Cost Analysis-CSP.pdf

[20] Muñoz, J., Martinez-Val, J. and Ramos, A. (2011) Thermal Regimes in SolarThermal Linear Collectors. Solar Energy, 85, 857-870.

https://doi.org/10.1016/j.solener.2011.02.004

[21] Abbas Cámara, R., Muñoz Antón, J. and Martínez-Val Peñalosa, J.M. (2012) Steady-State Thermal Analysis of an Innovative Receiver for Linear Fresnel Reflectors. Applied Energy, 92, 503-515. https://doi.org/10.1016/j.apenergy.2011.11.070

[22] Muñoz-Antón, J., Martínez-Val, J.M., González-Portillo, L.F., Cano, J. and Millán, J.S. (2019) Experimental Facility for a New Thermal-Solar Field Configuration: The Rotatory Fresnel Collector or Sundial. AIP Conference Proceedings, 2126, Article ID: 060007. https://doi.org/10.1063/1.5117593

[23] Martinez-Val Penalosa, J.M., Munoz Anton, J., Abbas Camara, R., Piera Carrete, M., Rovira De Antonio, A.J. and Montes Pita, M.J. (2017) Dispositivo para combar placas planas y procedimiento de uso.

[24] Martinez-Val Penalosa, J.M., Munoz Anton, J., Abbas Camara, R., Piera Carrete, M., Montes Pita, M.J. and Rovira De Antonio, A.J. (2015) Dispositivo rotatorio horizontal de concentración de la radiación solar.

[25] Tecnogetafe (2020) Tecnogetafe. https://www.tecnogetafe.es/en

[26] Fundación Fomento Innovación Industrial (2020) Fundación para el fomento de la innovación industrial. http://www.f2i2.net

[27] García Garrido, S. (2011) Ingeniería de centrales termosolares CCP. Renovetec, Madrid. http://santiagogarciagarrido.com/index.php/64-ingenieria-termosolar

[28] Renovetec (12/04/16) Presupuesto de construcción de una central termosolar. https://www.youtube.com/watch?v=N9BZDnppNM8

[29] Abbas, R., Montes, M.J., Rovira, A. and Martínez-Val, J.M. (2016) Parabolic Trough Collector or Linear Fresnel Collector? A Comparison of Optical Features Including Thermal Quality Based on Commercial Solutions. Solar Energy, 124, 198-215. https://doi.org/10.1016/j.solener.2015.11.039 


\section{Acronyms}

The following nomenclature and abbreviations are used in this manuscript:

CSP Concentrating Solar Power

STE Solar Thermal Energy (Plants)

TES Thermal Energy Storage

BOP Block of Power

$\mathrm{SF}$ Solar Field

HCF Heat Carrier Fluid

IHX Internal Heat Exchanger

PTC Parabolic Trough Collector

RFC Rotary Fresnel Collector 